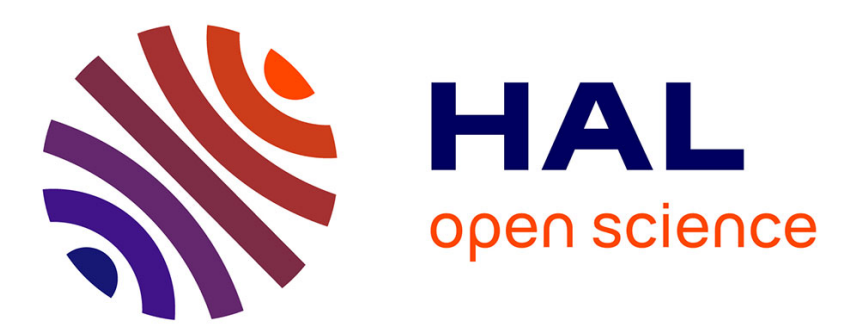

\title{
Comparison of energy-, pressure- and enthalpy-based approaches for modeling supercritical flows
}

Guilhem Lacaze, Schmitt Thomas, Anthony Ruiz, Joseph C Oefelein

\section{To cite this version:}

Guilhem Lacaze, Schmitt Thomas, Anthony Ruiz, Joseph C Oefelein. Comparison of energy-, pressureand enthalpy-based approaches for modeling supercritical flows. Computers and Fluids, 2019, 181, pp.35-56. 10.1016/j.compfluid.2019.01.002 . hal-02409206

\section{HAL Id: hal-02409206 \\ https://hal.science/hal-02409206}

Submitted on 18 Dec 2020

HAL is a multi-disciplinary open access archive for the deposit and dissemination of scientific research documents, whether they are published or not. The documents may come from teaching and research institutions in France or abroad, or from public or private research centers.
L'archive ouverte pluridisciplinaire HAL, est destinée au dépôt et à la diffusion de documents scientifiques de niveau recherche, publiés ou non, émanant des établissements d'enseignement et de recherche français ou étrangers, des laboratoires publics ou privés. 


\title{
Comparison of energy-, pressure- and enthalpy-based approaches for modeling supercritical flows
}

\author{
Guilhem Lacaze $^{\mathrm{a}, *}$, Thomas Schmitt', Anthony Ruiz ${ }^{\mathrm{a}}$, Joseph C. Oefelein ${ }^{\mathrm{c}}$ \\ ${ }^{a}$ Sandia National Laboratories, Livermore, CA, 94550, USA \\ ${ }^{b}$ Laboratoire EM2C, CNRS, Ecole CentraleSupélec, Université Paris-Saclay, 3 rue Joliot \\ Curie, 91192 Gif-sur-Yvette cedex, France \\ ${ }^{c}$ Daniel Guggenheim School of Aerospace Engineering, Georgia Institute of Technology, \\ Atlanta, GA, 30332, USA
}

\begin{abstract}
At supercritical conditions, thermodynamics may become strongly nonlinear which is reflected by large thermodynamic Jacobian values. This means that small variations in density, momentum or energy can result in large pressure perturbations. Because of such nonlinearities, simulations of high-pressure flows are subject to stability issues when the conservative form of the Navier-Stokes system is employed. In high-Reynolds number simulations, transported quantities are altered by numerical scheme errors, stabilization methods and filtering procedures. These alterations affect density and energy independently and may lead to significant pressure oscillations due to the nonlinear behavior of the equation of state. The objective of the present work is to compare three methods based on different sets of transport equations, to measure their impact on pressure and mixing temperature. A classical fully-conservative approach based on the transport of internal energy is compared to two quasi-conservative methods: a pressure-based and an enthalpy-based formulation. A suite test cases of increasing complexity is employed to expose the main differences between these approaches, at conditions relevant for engineering applications. The analysis first confirmed that the energy-based formulation is prone to artificial pressure fluctuations caused by the interaction between thermodynamic nonlinearities
\end{abstract}

\footnotetext{
* Corresponding author

Email address: guilhem.lacaze@gmail.com ( Guilhem Lacaze )
} 
and the numerical dissipation introduced by the stabilization scheme. It is also demonstrated that despite the stability properties of the pressure-based formulation, the mixing temperature is not properly captured since the pressure equation is not used in its conservative form. The enthalpy-based approach however, offers an improved representation of the mixing thermodynamic state while avoiding non-physical pressure variations.

Keywords: Supercritical Flow, Spurious Oscillations, Numerical Stability, Numerical Dissipation, Pressure-Based Equations, Enthalpy-Based Equations

\section{Introduction}

Propulsion and transportation systems (e.g., liquid rocket, gas turbine, and diesel engines) rely on high-pressure operating conditions to enhance performance. In these devices, the fuel or oxidizer are injected as a liquid into a 5 highly turbulent gaseous environment for efficient mixing. At low pressure, this process induces classical atomization coupled with evaporation where the interface between the liquid and the gas has a thickness on the order of the molecular scale. At high pressure, the gas-liquid interactions can dramatically change. Molecules of the gas are packed tighter against the liquid interface and inter-molecular forces between "gas" and "liquid" phases are no longer negligible. This yields a diminution of the surface-tension and a thickening of the interface until it becomes diffuse and continuous $[1,2]$. Mixing between dense and light fluids does not result in the creation of drops and ligaments. Instead, diffusion dominated mixing occurs in presence of a large density gradient. A comprehensive review on the effect of high-pressure nonlinearities in practical systems has been performed by Oschwald et al. [3]. For a pure species, this change occurs when the pressure rises above the critical pressure. Thermodynamic conditions are then called supercritical with respect to pressure. In the case of a mixture, the change in the thermodynamics is more difficult to predict since the transition locus cannot be directly determined from the critical points of the species of the mixture. Dahms and Oefelein [4] used linear gradient the- 
ory to investigate this process in propulsion applications. They showed that residual surface tension can still be measured above the critical points of the species in presence (also observed experimentally, for example by Woodward 25 et al. [5]), and that high-pressure diffusion dominated mixing can be expected in real engineering systems. In these systems, the term transcritical mixing is often used to characterize the dynamics that occurs between two fluids, one at subcritical temperature and the other one at supercritical temperature, since conditions cross the pseudo-boiling line [3].

The lack of a comprehensive understanding of mixing under supercritical conditions has stimulated significant modeling efforts to complement experimental and theoretical research. A major difficulty, however, is the capability of numerical solvers to maintain accuracy and stability in regions of large gradients in density and thermodynamic quantities. This aspect is the focal point

35 of the present work. Because of strong thermodynamic non-linearities in the vicinity of pseudo boiling line, small variations of density and/or energy may generate pressure oscillations. In high-Reynolds and high-pressure simulations, stabilization schemes are often required to handle strong field gradients. Density, energy and other transported quantities are then independently dissipated, 40 and may become "thermodynamically misaligned". This means that due to nonlinearities in the equation of state, the reconstructed pressure $P\left(\bar{\rho}, \overline{\rho e}, \overline{\rho Y_{k}}\right)$ may differ from the real physical pressure (this issue is illustrated in Section 2) and may result in artificial pressure variations. These non-physical oscillations can deteriorate the predictions accuracy or lead to the divergence of the calculation.

${ }_{45}$ This has been previously reported by Hickey et al. [6], Terashima and Koshi [7], Kawai et al. [8] and Schmitt et al. [9]. In Reynolds Average Navier Stokes (RANS) simulations, gradients are diffused and well resolved which minimizes the problem $[10,11,12,13,14,15,16,17]$. In Direct Numerical Simulations (DNS), grid resolution is selected to resolve all scales, which also circumvents 50 the issue. For example, Bellan and co-workers have studied temporal mixing layers under supercritical conditions using sixth- to eighth- order accurate central finite differences for spatial derivative along with a filtering step (eighth- to 
tenth-order $)[18,19,20,21,22,23]$. Other researchers have employed similar approaches $[24,25,26]$.

Large Eddy Simulation (LES) applied to high-pressure systems is more prone to this problem since the Reynolds numbers of simulated flows are usually greater than in DNS and coarser grids are employed. Different strategies have been tried to mitigate the stability issue. Many researchers have used dense grid resolution to limit discretization errors in gradient regions. This approach requires large computational resources and are usually applied to small scale computational domains such as splitter plates [27, 28], supercritical jets [29, 30], mixing layers $[31,32]$ and single rocket injectors $[33,34,35]$. Others have employed various dissipation techniques to diffuse gradients and attain sufficient resolution in gradient zones. Dissipative convective schemes have been used, several of them derived from shock-capturing approaches. In the simulations of Oefelein et al. [27] and more recently in [36, 37, 38, 30], spatial discretization is based on a high-order flux differencing technique coupled with a total-variationdiminishing (TVD) scheme. Matsuyama et al. [39, 34] used second- and thirdorder MUSCL schemes and Smith et al. [40] stabilized their simulation with a second-order approximate Riemann solver. Hickey et al. [6] used a second-order accurate Essentially Non-Oscillating (ENO) scheme which was switched to a first-order upwind scheme in gradient regions. Similar approach have been used by Park et al. [41], Muller et al. [42] and Matheis et al. [43]. Dissipation was also introduced via artificial diffusion schemes. This latter approach has been employed by Meng and Yang [44] and recently by Masquelet et al. [45], Yang et al. [46, 47]. More advanced artificial viscosity methods have also been derived by Terashima et al. [48] and Zong et al. [49, 50, 51, 52, 28] which combines second and fourth order dissipation operators as well as bulk viscosity. Recently, Schmitt et al. $[9,53,54]$ showed that to limit the creation of nonphysical oscillations, the thermodynamic balance between density, species momentum and energy must be preserved. To do so, they derived a non-conservative form that calculates the amount of dissipation needed on energy, given the dissipation on density and species mass-fraction to obtain $P_{\text {mechanical }} \approx P_{\text {eos }}\left(\bar{\rho}, \overline{\rho e}, \overline{\rho Y_{k}}\right)$, 
where $P_{\text {mechanical }}$ is the pressure used in the transport equations and $P_{\text {eos }}$ is the pressure derived from the thermodynamic scheme. This method has recently been used in multiple rocket-like configurations to investigate combustion and acoustic instabilities $[9,55,56,57]$. Ma et al. [58] have extended the doubleflux method to real fluid thermodynamics by imposing the same heat capacity in adjacent cells when fluxes are constructed. This approach is no longer energy conservative but eliminates non-physical pressure oscillations when large gradients are present in the flow. Other groups have tabulated thermodynamic quantities and derivatives to eliminate errors created when thermodynamics parameters and Jacobians are estimated numerically.

This approach provides some improvements but does not totally alleviate the ${ }_{95}$ issue $[29,59,8]$. Taşkinoğlu and Bellan [60] and Selle et al. [61] have conserved all terms usually neglected when the Navier-Stokes equation system is filtered to build the LES transport framework. They identified additional subgrid-scale terms to correct the error produced when pressure is evaluated from filtered quantities. However, they showed that the accuracy of the subgrid-scale models for pressure rapidly deteriorates when the mesh is coarsened compared to DNS. An efficient approach to eliminate the spurious pressure oscillations is to directly transport pressure through a quasi-conservative system. Karni [62] pioneered this research direction and successfully implemented a pressure-based (PB) solver for multicomponent gaseous flow. Terashima and Koshi [7, 63] extended this approach to supercritical conditions. These developments have since then been used by other researchers $[64,65]$. A recent energy conservative strategy for the Van Der Waals equation of state has been proposed by Pantano et al. [66]. In this work a new variable that separates the evolution of the nonlinear part of the density in the equation of state is added to the flow model resulting in an oscillation free solution.

Despite the number of studies dedicated to suppressing nonphysical pressure oscillations at high-pressure conditions, a comprehensive comparison between conservative and non-conservative approaches still needs to be performed. Terashima et al. [48], Terashima and Koshi [7] and Kawai et al. [8] only used 
pure advection tests to compare classical (conservative) and pressure-based approaches. Their conclusions were that less dissipation is needed with the latter since simulations were globally more stable. Matheis et al. [65] used a fully conservative approach and a pressure-based formulation to simulate a turbulent nitrogen jet, mixing into a quiescent gaseous hydrogen environment. Given the complexity of the configuration, only general observations were made. Less pressure fluctuations were observed when the pressure-based formulation was employed, along with a delay in the destabilization of the dense core. Significant temperature differences were also detected but the origin was not identified.

The purpose of the present work is to perform a systematic comparison between three different approaches that differ by the set of transported quantities. Particular emphasis is placed on their impact on pressure and mixing temperature. The classical fully-conservative "energy-based" approach (EB) is considered as a reference where density, energy, momentum and species are transported. The second approach is a pressure-based approach (PB) where density, pressure, momentum and species are the transported quantities. Finally, a new approach is proposed, called enthalpy-based approach (HB), where two transport equations for pressure and total energy are combined to estimate the spatio-temporal evolution of enthalpy, along with equations on density, momentum and species. These three approaches are tested within the same solver using the same numerical methods and physical models. This is accomplished using a sequence of canonical cases that isolate specific aspects of numerics and turbulent mixing under high-Reynolds numbers and elevated pressures.

The paper is organized as follows. Section 2 provides an explanation of the numerical challenges present in high-pressure, high- density flow simulations. 40 This is followed by Section 3 which describes the three approaches and provides details on their derivations. The same section presents the associated models and numerics of the flow solver. Finally, Section 4 presents comparisons of the three approaches on canonical, advection, one-dimensional shock, and vortex-interface test cases. The analysis concludes on a more realistic case of a $R e=500,000$ mixing layer mimicking flow conditions present at the exit of a rocket coaxial 
injector.

\section{Thermodynamic non-linearities and numerical challenges}

At elevated pressures intermolecular forces become significant, which modifies the relationship between pressure, density, and temperature. In order to capture these "real fluid" effects, van der Waals was the first to try to incorporate the impact of intermolecular forces into the equation of state [2]. Subsequently, other groups proposed corrections and improvements to generalize the approach. Currently, cubic equations of state (EoS) are widely used in computational fluid dynamics due to their computational affordability and acceptable accuracy. For the present study, the Peng-Robinson [67] equation has been selected since it offers satisfactory accuracy for the $\mathrm{O}_{2}-\mathrm{H}_{2}$ system. Figure 1 presents the thermodynamic scheme predictions of density, heat capacity at constant pressure and speed of sound compared to the reference database from NIST [68] for pure oxygen at $P=100 \mathrm{bar}$, and over the temperature range $[150 K-300 K]$. A maximum error of $12 \%$ is observed on density, and $11 \%$ on speed of sound. Since the present study focuses more on the behavior of the equation of state than on the absolute precision, this level of accuracy is considered acceptable. Also, the same equation of state is used in all the approaches tested in this work. Note, however, that any other EoS could be used for the present analysis.

The Peng Robinson EoS has the form:

$$
P=\frac{R T}{V-B_{m}}-\frac{A_{m}}{V^{2}+2 B_{m} V-B_{m}^{2}},
$$

where $R$ is the ideal-gas constant, $V$ the molar volume, $T$ and $P$ the temperature and the pressure, respectively. The coefficients $A_{m}$ and $B_{m}$ account for attraction and repulsion effects among molecules. They are calculated using a set of nonlinear mixing-rules [2, Chapter 4] given by

$$
A_{m}=\sum_{k=1}^{N s} \sum_{l=1}^{N s} X_{k} X_{l} \sqrt{A_{k} A_{l}} k_{k l}, \quad B_{m}=\sum_{k=1}^{N s} X_{k} B_{k}
$$




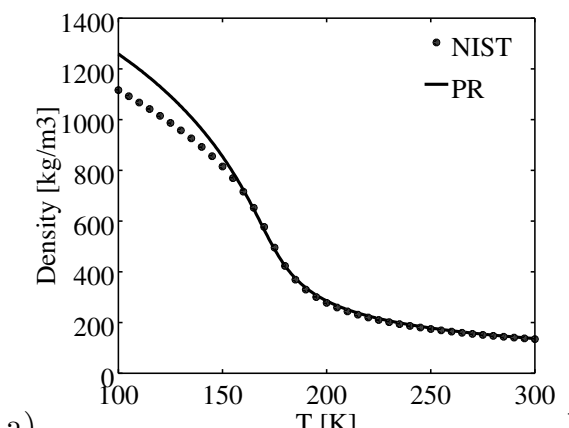

a)
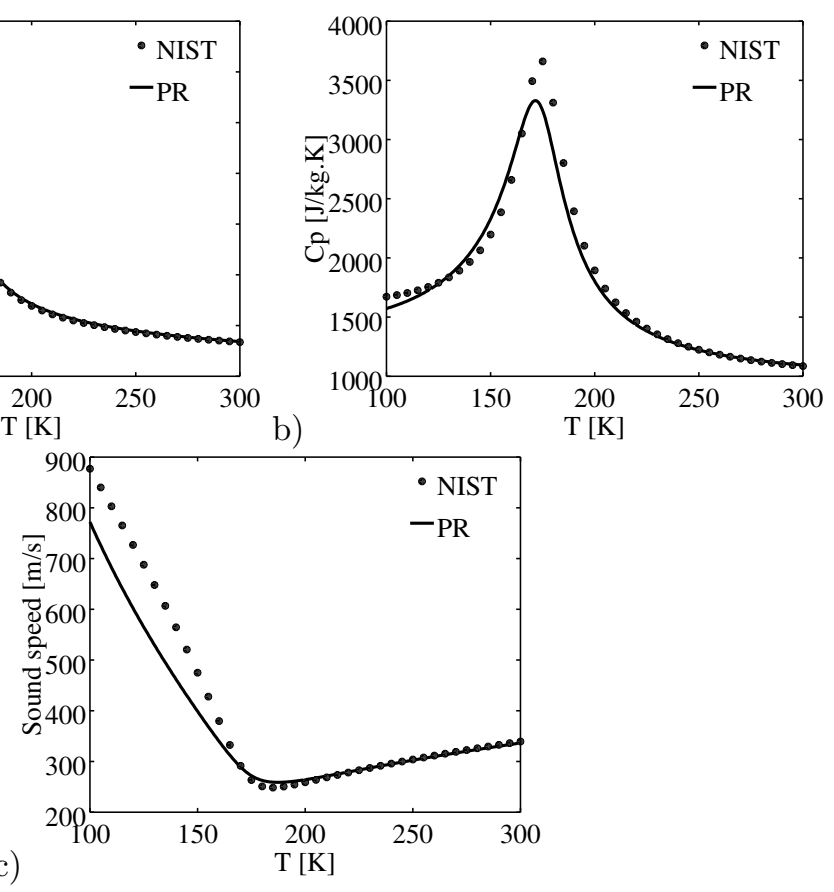

Figure 1: Thermodynamic predictions of the Peng-Robinson equation of state for pure oxygen at $P=100$ bar: (a) density, (b) heat capacity at constant pressure and (c) speed of sound. Predictions of the Peng-Robinson (PR) equation of state (line) are compared to the NIST database (symbols) [68].

where

$$
\begin{aligned}
A_{k} & =0.457236 \frac{\left(R T_{c, k}\right)^{2}}{P_{c, k}}\left[1+C_{k}\left(1-\sqrt{T / T_{c, k}}\right)\right]^{2}, \\
B_{k} & =0.077796 R T_{c, k} / P_{c, k} \\
C_{k} & =0.37464+1.54226 \omega_{k}-0.2699 \omega_{k}^{2} .
\end{aligned}
$$

In the expression above, $X_{k}$ is the molar fraction of species $k, k_{k l}$ is the binary interaction parameter between species $k$ and $l$ and $\omega_{k}$ is the acentric factor of species $k . P_{c, k}$ and $T_{c, k}$ are the critical pressure and temperature of species $k$, respectively.

A two step approach is used to determine the thermodynamic properties 175 at relevant conditions. First, the mixture properties are evaluated at the temperature of interest and at a reference pressure, using the corresponding states 
methodology. Departure functions are then applied to obtain the mixture state at the desired pressure [2]. These functions are exact relations derived from Maxwell's relations and make full use of the real mixture $p-v$ - $T$ path dependencies dictated by the equation of state. The sensible enthalpy $h(T, \rho)$ and heat capacity at constant pressure $C_{p}(T, \rho)$ are estimated by

$$
\begin{aligned}
h(T, \rho)-h^{\circ}(T) & =\int_{P^{\circ}}^{P}\left[\frac{1}{\rho}-\frac{T}{\rho^{2}}\left(\frac{\partial \rho}{\partial T}\right)_{P, Y_{k}}\right]_{T} d P \\
C_{p}(T, \rho)-C_{p}^{\circ}(T) & =-\int_{\rho^{\circ}}^{\rho}\left[\frac{T}{\rho^{2}}\left(\frac{\partial^{2} P}{\partial T^{2}}\right)_{\rho}\right]_{T} d \rho+\frac{T}{\rho^{2}} \frac{\left(\frac{\partial^{2} P}{\partial T^{2}}\right)_{\rho}}{\left(\frac{\partial P}{\partial \rho}\right)_{T}}
\end{aligned}
$$

where the superscript (o) represents the reference state. Derivatives in Eq. 6 and 7 are evaluated analytically from the equation of state. Standard state properties are obtained using the databases developed by Gordon and McBride [69] and Kee et al. [70].

It is useful to establish the sensitivity of real fluid thermodynamics in the context of computational fluid dynamics. This is accomplished by investigating the sensitivity of the equation of state (Peng-Robinson) with respect to small perturbations of density and energy.

In thermodynamic space and for a pure species, the variation of pressure depends on the variations in density and sensible energy:

$$
d P=(\partial P / \partial \rho)_{\rho e} d(\rho)+(\partial P / \partial \rho e)_{\rho} d(\rho e) .
$$

The Jacobians (see Appendix A for the derivation) are:

$$
\begin{aligned}
& (\partial P / \partial \rho e)_{\rho}=-\frac{\rho_{T}}{\rho C_{v} \rho_{P}}, \\
& (\partial P / \partial \rho)_{\rho e}=\frac{\rho C_{p}+h \rho_{T}}{\rho C_{v} \rho_{P}},
\end{aligned}
$$

where $C_{v}$ and $C_{p}$ are the heat capacities at constant volume and constant pressure, respectively. $h$ is the sensible enthalpy, $\rho_{p}$ and $\rho_{T}$ are the derivatives of density with respect to pressure and temperature. Note that in a flow, momentum also has an impact on pressure. By adding the contribution of kinetic 
energy $\left(e_{c}=1 / 2\left(U_{i} U_{i}\right)\right)$ to Eq. 8 , one can easily obtain:

$$
d P=-\frac{\rho_{T}}{\rho C_{v} \rho_{P}} d\left(\rho e_{t}\right)+\left[\frac{\rho C_{p}+\rho_{T}\left(h_{s}-e_{c}\right)}{\rho C_{v} \rho_{P}}\right] d \rho+\frac{\rho_{T}}{\rho C_{v} \rho_{P}} U_{i} d\left(\rho U_{i}\right),
$$

Figure 2 presents the evolution of density and the two Jacobian terms described in Eq. 9 and 10 for pure oxygen over ranges of pressure and temperature of interest for rocket engines (i.e., above critical critical pressure of oxygen: $P_{c}^{O_{2}}=$ $50.4 b a r$ ). One can observe that in the vicinity of the pseudo boiling line (where density varies significantly) both Jacobian terms change dramatically. This means that small variations in density and energy may result in large changes in pressure.

The impact of these non-linearities can be illustrated by a simple numerical experiment. A temperature variation (hyperbolic tangent function) is used to generate gradients in density and energy for pure oxygen at a pressure of 150bar. Asymptotic temperature values are initially set to $150 K$ and $300 K$, respectively. These values have been chosen to be on either side of the pseudo-boiling line at the considered pressure. For pure oxygen at 150 bar, the pseudo boiling point (maximum of heat capacity) is at $T=185 K \quad[17,16]$ (note that the critical temperature of oxygen is $\left.T_{c}^{O_{2}}=154.6 \mathrm{~K}\right)$. This results in a high density difference and positions the gradient in the high-Jacobian region in thermodynamic space. To simulate the alteration of transported quantities caused by numerical errors or stabilization methods, density $(\rho)$ and energy ( $\rho e)$ are filtered using Gaussian filters of different kernel widths. Note that the same analysis could be conducted by adding noise to the data (in the gradient region). Subsequently, pressure and temperature are re-evaluated based on filtered density and energy: $P=f(\bar{\rho}, \overline{\rho e})$ and $T=g(\bar{\rho}, \overline{\rho e})$, where $f$ and $g$ are relationships representing the impact of the equation of state.

Figure 3 shows filtered density and energy fields, and the resultant pressure and temperature. Temperature is fairly insensitive to the filtering operation. Pressure, however, is significantly impacted and presents a large non-physical decrease in the gradient region. This shows that in the context of an equation system based on density and energy, small variations of the transported quan- 


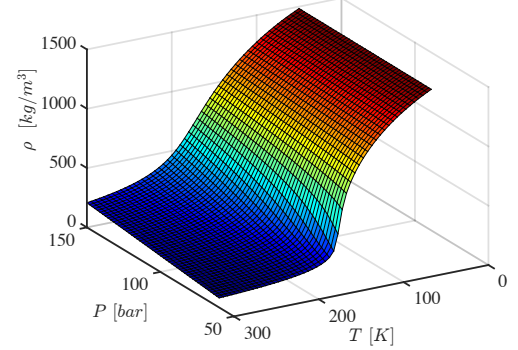

a)

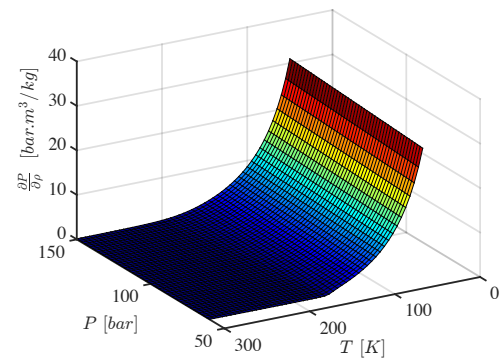

b)

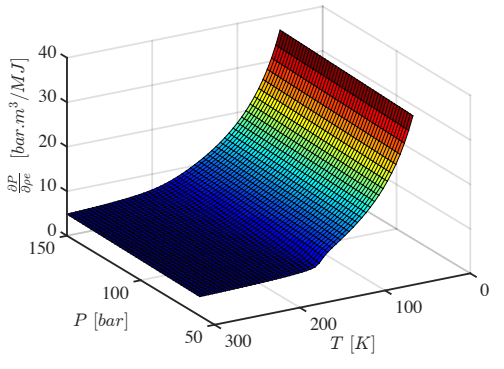

c)

Figure 2: Thermodynamic sensitivity at elevated pressure for pure oxygen in pressuretemperature space $([60-150]$ bar and $[60-300] \mathrm{K}):(\mathrm{a})$ density, (b) Jacobian term: $(\partial P / \partial \rho)_{\rho e}$ and (c) Jacobian term: $(\partial P / \partial \rho e) \rho$.

tities introduced by the numerical approach may result in significant pressure oscillations. These variations can be introduced by an explicit filtering step such as those used to remove high-frequency oscillations, numerical errors or 

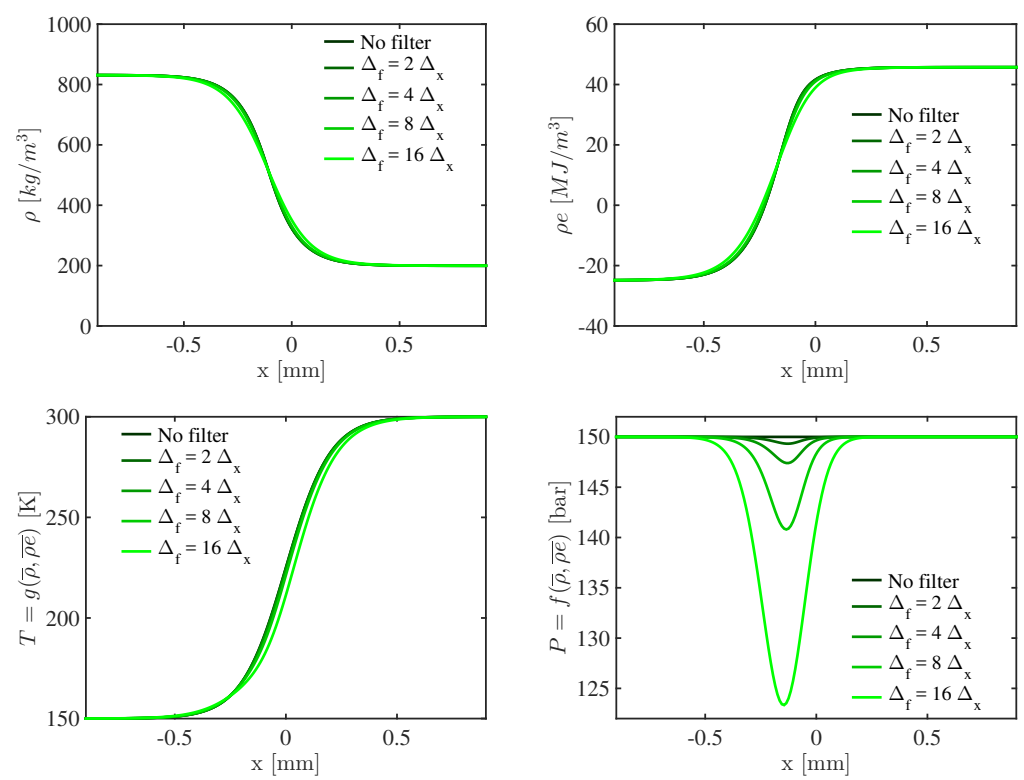

Figure 3: Impact of filtering on the estimation of pressure and temperature in a transcritical case (pure oxygen, $150 K<T<300 K$ and $P=150 \mathrm{bar}$ ). $\Delta_{f}$ is the filter size in grid spacing unit $\Delta_{x}$.

the dissipation of a stabilization method.

This issue however is only significant close to the pseudo boiling line where the equation of state presents strong nonlinearities. If the same numerical experiment is replicated at the same pressure but in a temperature range where Jacobians have smaller values, the reconstructed pressure exhibits a different behavior. For example, Fig. 4 shows the same filtering test over the range $500 K<T<1000 K$ at $P=150$ bar. In this region, the equation of state presents a quasi-linear behavior and the filtering procedure does not introduce large deviations in pressure.

This illustration shows why in the vicinity of the pseudo-boiling line, an approach based on the transport of density and energy is prone to spurious pressure oscillations if artificial stabilization is required in gradient regions.

To circumvent this issue, an efficient strategy is to replace the energy equation by a pressure equation. While this approach is not conservative with 

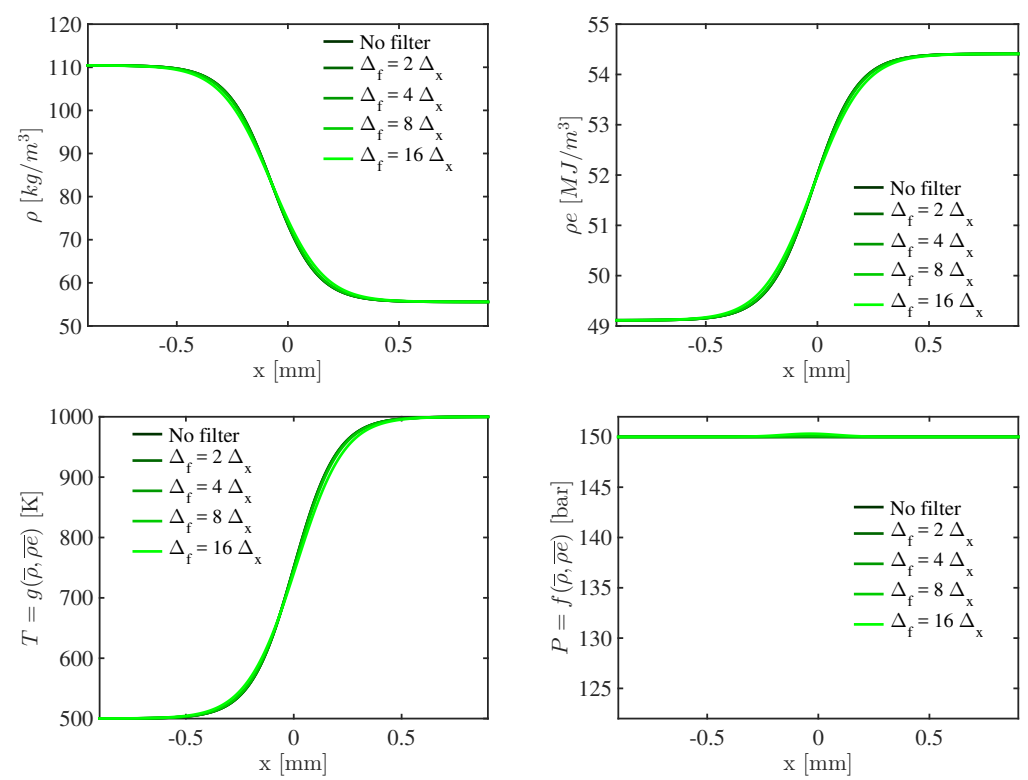

Figure 4: Impact of filtering on the estimation of pressure and temperature in a temperature range where non-linear effects are not significant (pure oxygen, $500 K<T<1000 K$ and $P=150$ bar $). \Delta_{f}$ is the filter size in grid spacing unit $\Delta_{x}$.

respect to energy, it suppresses unphysical pressure oscillations [8]. Another strategy, explored in this paper, is to combine energy-based and pressure-based approaches to retain energy conservation while avoiding spurious pressure oscillations due to the non-linearity of the equation of state. These methods are described in the next section.

\section{Theoretical and numerical approaches}

Three distinct approaches have been tested in the present investigation, The fully conservative Navier-Stokes system based on the transport of total energy is first introduced in Section 3.1. A pressure-based method is described in Section 3.2. As suggested by [7], this quasi-conservative method allows avoiding non-physical pressure disturbances under transcritical conditions when used with non-linear thermodynamics (in flows without shocks), but suffers from inaccuracy in the estimation of temperature and other thermodynamic variables. 
To improve this aspect, one can employ a quasi-conservative enthalpy-based approach, presented here in Section 3.3.

\subsection{Energy-based formulation (EB)}

The energy-based (EB) formulation involves the classical Navier-Stokes system of equations used to transport the conservative variables of density $(\rho)$, momentum $\left(\rho \mathbf{U}=\rho\left(U_{x}, U_{y}, U_{z}\right)^{T}\right)$, species mass fractions $\left(\rho Y_{k}\right.$, where $k$ is the index of $k^{t h}$ species of the mixture) and total energy $\left(e_{t}=h_{t}-p / \rho=e_{s}+1 / 2 \mathbf{U} \cdot \mathbf{U}\right.$, where $h_{t}$ is the total enthalpy and $e_{s}$ is the sensible energy The governing system is given by

$$
\begin{gathered}
\frac{\partial \rho}{\partial t}+\nabla \cdot(\rho \mathbf{U})=0 \\
\frac{\partial}{\partial t}\left(\rho e_{t}\right)+\nabla \cdot\left(\left(\rho e_{t}+P\right) \mathbf{U}\right)=\nabla \cdot\left(-\mathbf{q}_{e}+\tau \cdot \mathbf{U}\right), \\
\frac{\partial}{\partial t}(\rho \mathbf{U})+\nabla \cdot[\rho \mathbf{U} \otimes \mathbf{U}+P \mathbf{I}]=\nabla \cdot \tau \\
\frac{\partial}{\partial t}\left(\rho Y_{k}\right)+\nabla \cdot\left(\rho Y_{k} \mathbf{U}\right)=-\nabla \cdot \mathbf{q}_{k} .
\end{gathered}
$$

In this system,

$$
\tau=\mu\left[-\frac{2}{3}(\nabla \cdot \mathbf{U}) \mathbf{I}+\left(\nabla \mathbf{U}+\nabla \mathbf{U}^{T}\right)\right] .
$$

represents the viscous stress tensor, where $\mu$ is the dynamic viscosity and $P$ is the pressure. $\mathbf{q}_{k}$ represents the mass diffusion fluxes of the $k^{t h}$ species, and contains the velocity correction $U_{\text {corr }}$ (to ensure global mass conservation) resulting from the Hirschfelder and Curtiss approximation [71]:

$$
\mathbf{q}_{k}=-\rho\left(D_{k} \frac{W_{k}}{W} \nabla X_{k}-Y_{k} U_{c o r r}\right)
$$

where $X_{k}, D_{k}$ and $W_{k}$ are the molar fraction, the molar diffusivity and the molar mass of the $k^{t h}$ species, respectively. $W$ is the molar mass of the mixture. 
The term $\mathbf{q}_{e}$ is the energy diffusion flux expressed as:

$$
\mathbf{q}_{e}=-\lambda \nabla T+\sum_{k=1}^{N_{s}} \mathbf{q}_{k} h_{k},
$$

where $\lambda$ is the thermal conductivity of the mixture, $T$ the temperature and $h_{k}$ the enthalpy of the $k^{t h}$ species. Note that Soret and Dufour effects are neglected.

At supercritical pressures, molecular transport properties are evaluated in a manner analogous to the thermodynamic properties previously presented in Section 2. Viscosity and thermal conductivity are obtained using the extended corresponding state methodologies developed by Ely and Hanley [72]. Mass and thermal diffusion coefficients are obtained using the approach from Hirschfelder et al. [1] and Takahashi [73]. The present framework can handle a wide range of pressure and temperature where multicomponent and/or preferential diffusion processes are present. The mixture diffusion coefficient for species $k, D_{k}$ is computed as a function of the matrix of binary diffusion coefficients $D_{i j}$, as obtained from kinetic theory $[1,74]$ :

$$
D_{k}=\frac{1-Y_{k}}{\sum_{j \neq k}^{N_{s}} X_{j} / D_{j k}}
$$

Thermodynamic properties, pressure and temperature are evaluated thanks to a conventional Newton iterative procedure. First, pressure is calculated from the equation of state using the transported density and an initial guess on temperature. The thermodynamic energy is then computed, and compared to the transported energy. Temperature is updated using this difference and the heat capacity at constant volume. This procedure is repeated until the error on energy drops below a threshold set by the user (set here at $10^{-10}$, sensitivity analysis has shown no degradation of accuracy for values below $10^{-8}$ ). Once pressure and temperature are found, all other thermodynamic and transport quantities are calculated via the equation of state.

\subsection{Pressure-based formulation (PB)}

In this subsection, the derivation of the pressure evolution equation is presented. Only the main steps of the derivation are listed here for sake of brevity. 
Additional details on the derivation are provided in Appendix B. The static pressure equation is derived from the Gibbs-Duhem equation [2]:

$$
\frac{D P}{D t}=\left(\frac{\partial P}{\partial \rho}\right)_{e, Y_{k}} \frac{D \rho}{D t}+\left(\frac{\partial P}{\partial e}\right)_{\rho, Y_{k}} \frac{D e}{D t}+\sum_{k=1}^{N_{s}}\left(\frac{\partial P}{\partial Y_{k}}\right)_{e, \rho} \frac{D Y_{k}}{D t}
$$

where $e$ is sensible energy, with $D \phi / D t=\partial \phi / \partial t+\mathbf{U} \cdot \nabla \phi$ the total derivative for any quantity $\phi$. Using the transport equations previously introduced in Section 3.1, we obtain the transport equation for pressure:

$$
\begin{aligned}
\frac{\partial P}{\partial t}+\mathbf{U} \cdot \nabla P= & \left(\left(\frac{\partial P}{\partial \rho}\right)_{e, Y_{k}}+\frac{P}{\rho^{2}}\left(\frac{\partial P}{\partial e}\right)_{\rho, Y_{k}}\right)(-\rho \nabla \cdot \mathbf{U}) \\
& +\left(\frac{\partial P}{\partial e}\right)_{\rho, Y_{k}}\left(\frac{1}{\rho}\left(-\nabla \cdot \mathbf{q}_{e}+\tau \nabla \cdot \mathbf{U}\right)\right) \\
& +\sum_{k=1}^{N_{s}}\left(\frac{\partial P}{\partial Y_{k}}\right)_{e, \rho}\left(\frac{1}{\rho}\left(-\nabla \cdot \mathbf{q}_{k}\right)\right),
\end{aligned}
$$

Using the relation B.12 of Appendix B the derivatives introduced above can be explicitly expressed:

$$
\begin{aligned}
& \left(\frac{\partial P}{\partial \rho}\right)_{e, Y_{k}}=c^{2}\left[1+\frac{P \rho_{T}}{C_{p} \rho^{2}}\right] \\
& \left(\frac{\partial P}{\partial e}\right)_{\rho, Y_{k}}=-\frac{\rho_{T} c^{2}}{C_{p}} \\
& \left(\frac{\partial P}{\partial Y_{k}}\right)_{e, \rho}=\frac{c^{2}}{C_{p}}\left[\rho_{T} h_{Y_{k}}-C_{p} \rho_{Y_{k}}\right]
\end{aligned}
$$

where

$$
\begin{aligned}
\rho_{T} & =\left(\frac{\partial \rho}{\partial T}\right)_{P, Y_{k}}, \quad \rho_{P}=\left(\frac{\partial \rho}{\partial P}\right)_{T, Y_{k}}, \quad \rho_{Y_{k}}=\left(\frac{\partial \rho}{\partial Y_{k}}\right)_{P, T}, \\
h_{Y_{k}} & =\left(\frac{\partial h}{\partial Y_{k}}\right)_{P, T} \text { and, } \quad c^{2}=\left(\frac{\partial P}{\partial \rho}\right)_{s, Y_{k}}=\frac{\gamma}{\rho_{P}} .
\end{aligned}
$$

Note that the derivatives $\rho_{T}, \rho_{P}, \rho_{Y_{k}}$ and $h_{Y_{k}}$ are analytically evaluated from the equation of state and $\gamma$ is the heat capacity ratio. Using these derivatives, Eq. 21 can be fully expanded and the system of governing of equations can be written as:

$$
\frac{\partial \rho}{\partial t}+\nabla \cdot(\rho \mathbf{U})=0
$$




$$
\begin{gathered}
\frac{\partial P}{\partial t}+\mathbf{U} \cdot \nabla P=-\rho c^{2} \nabla \cdot \mathbf{U}-\frac{c^{2} \rho_{T}}{\rho C_{p}}\left(-\nabla \cdot \mathbf{q}_{e}+\tau \nabla \cdot \mathbf{U}\right) \\
+\sum_{k=1}^{N_{s}} \frac{c^{2}}{\rho C_{p}}\left(\rho_{T} h_{Y_{k}}-\rho_{Y_{k}} C_{p}\right)\left(-\nabla \cdot \mathbf{q}_{k}\right), \\
\frac{\partial}{\partial t}(\rho \mathbf{U})+\nabla \cdot[\rho \mathbf{U} \otimes \mathbf{U}+P \mathbf{I}]=\nabla \cdot \tau, \\
\frac{\partial}{\partial t}\left(\rho Y_{k}\right)+\nabla \cdot\left(\rho Y_{k} \mathbf{U}\right)=-\nabla \cdot \mathbf{q}_{k} .
\end{gathered}
$$

Where $\mathbf{q}_{k}$ and $\mathbf{q}_{e}$ have been previously introduced in Eq. 17 and 18, respectively. Note that equation (29) is consistent with the simplified transport equation derived by Terashima and Koshi [7] given that:

$$
\frac{\alpha_{P}}{C_{v} \beta_{T} \rho}=\frac{\alpha_{P} \rho c^{2}}{C_{v} \gamma \rho}=\frac{\alpha_{P} c^{2}}{C_{p}}=-\frac{c^{2} \rho_{T}}{C_{P} \rho},
$$

where $C_{v}$ is the heat capacity at constant volume, $\alpha_{P}=1 / V(\partial V / \partial T)_{P}$ is the thermal expansion coefficient, and $\beta_{T}=-1 / V(\partial V / \partial P)_{T}$ is the isothermal compressibility coefficient.

Temperature, thermodynamic and transport quantities are evaluated using a similar Newton iteration method as in the EB approach. Here the method start from an initial guess on temperature, from which a thermodynamic density $\left(\rho_{\text {eos }}\right)$ is determined using the equation of state and the transported pressure. This density is compared to the transported density $\left(\rho_{\text {trans }}\right)$ and the difference is used to update temperature, using the increment:

$$
d T=\frac{\rho_{\text {trans }}-\rho_{\text {eos }}}{\rho_{T}},
$$

where $\rho_{T}$ is the derivative of density with respect to temperature which is analytically estimated from the roots of the equation of state. This procedure is iteratively solved until the error on density falls below a threshold set by the user $\left(10^{-10}\right.$ in the present study). All other properties are then calculated from $275 T$ and $\rho Y_{k}$. 


\subsection{Enthalpy-based formulation (HB)}

This approach is based on the transport of enthalpy instead of energy. The temporal evolution of enthalpy is described as the sum of the evolutions in pressure and energy. The objective is to use the oscillation-free transported pressure $P_{\text {trans }}$ as the mechanical pressure (used in momentum and energy equations), and to compute thermodynamic variables from the transported enthalpy (obtained by combining transported pressure and energy). This formulation provides the dynamic stability of a pressure-based method while estimating thermodynamics with a similar accuracy than the energy-based approach. This approach conserves total energy by construction since it is a transported quantity. The equation for total energy is the same as in the energy-based approach, with no additional source or sink terms, and all flux terms (viscous, inviscid, and pressure) are uniquely defined at each cell face, which guaranties discrete conservation in the present finite-volume formalism. However, pressure is obtained from a non conservative transport equation, which makes the approach only quasi-conservative as enthalpy is not exactly conserved.

The transported quantities are density $(\rho)$, momentum $(\rho \mathbf{U})$, species mass fractions $\left(\rho Y_{k}\right)$ and total enthalpy $\left(h_{t}\right)$. The governing system of the HB approach reads:

$$
\begin{aligned}
& \frac{\partial \rho}{\partial t}+\nabla \cdot(\rho \mathbf{U})=0, \\
& \frac{\partial \rho h_{t}}{\partial t}=\frac{\partial \rho e_{t}}{\partial t}+\frac{\partial P}{\partial t},
\end{aligned}
$$

which can be expended to:

$$
\begin{aligned}
\frac{\partial \rho h_{t}}{\partial t}= & -\nabla \cdot\left(\left(\rho e_{t}+P\right) \mathbf{U}\right)+\nabla \cdot\left(-\mathbf{q}_{e}+\tau \cdot \mathbf{U}\right) \\
& -\mathbf{U} \cdot \nabla P-\rho c^{2} \nabla \cdot \mathbf{U}-\frac{c^{2} \rho_{T}}{\rho C_{p}}\left(-\nabla \cdot \mathbf{q}_{e}+\tau \nabla \cdot \mathbf{U}\right) \\
& +\sum_{k=1}^{N_{s}} \frac{c^{2}}{\rho C_{p}}\left(\rho_{T} h_{Y_{k}}-\rho_{Y_{k}} C_{p}\right)\left(-\nabla \cdot \mathbf{q}_{k}\right),
\end{aligned}
$$


where $P$ is estimated using equation 29 .

$$
\begin{gathered}
\frac{\partial}{\partial t}(\rho \mathbf{U})+\nabla \cdot\left[\rho \mathbf{U} \otimes \mathbf{U}+P_{\text {trans }} \mathbf{I}\right]=\nabla \cdot \tau, \\
\frac{\partial}{\partial t}\left(\rho Y_{k}\right)+\nabla \cdot\left(\rho Y_{k} \mathbf{U}\right)=-\nabla \cdot \mathbf{q}_{k},
\end{gathered}
$$

As classically done, the thermodynamic state is calculated using a Newton iterative procedure. Here, the inputs of the thermodynamic scheme are the transported enthalpy $\left(h_{\text {trans }}=\left[(\rho e)_{\text {trans }}+P_{\text {trans }}\right] / \rho_{\text {trans }}\right)$ and density $\left(\rho_{\text {trans }}\right)$. The same relative threshold of $10^{-10}$ is used here, as in previous approaches.

The pressure used in the enthalpy-based systemis obtained via its own transport equation. This pressure is referred as mechanical pressure as it appears in momentum and energy equations. A thermodynamic pressure could also be estimated from density and enthalpy using the thermodynamic scheme. This pressure is not used since it suffers from non-physical oscillations in gradient regions due to the impact of numerical dissipation. In other regions of the flow, both pressures are identical.

\subsection{Numerical approach}

Calculations were performed using the numerical framework developed by Oefelein [36, 38]. For all three approaches, the same explicit four-stage RungeKutta temporal scheme $[75,76]$ has been used with a time-step restriction based on the Courant-Friedrichs-Lewy condition: $C F L_{\max }=0.5$. The governing systems are discretized on a staggered grid in generalized curvilinear coordinates in finite-volume form. Diffusive fluxes are estimated with a second-order accurate centered difference scheme. Convective fluxes are evaluated using a secondorder QUICK scheme (Quadratic Upstream Interpolation for Convective Kinematics) [77]. To ensure stability, the scheme transitions to a first-order upwind scheme [78] in gradient regions if spatial resolution is insufficient. A detailed description of the stabilization approach is outlined in Appendix C. Note that 
in the case of the PB and $\mathrm{HB}$ formulations, the gradient terms in the pressure equation are evaluated with centered operators. The residual terms coming from the stabilization of the conserved quantities have not been incorporated. These terms are described in Appendix D. In this manner, spurious pressure oscillations caused by the numerical stabilization scheme are avoided, which makes the PB and HB approaches dynamically more stable than the energybased method. However, because of this modification, the pressure equation is no longer conservative. This is why the present pressure- and enthalpy-based formulations are not conservative.

\section{Results and discussion}

Four benchmark tests of increasing complexity have been considered for the present analysis. All cases involved oxygen and hydrogen at the conditions previously studied by Oefelein [38] and Ruiz et al. [79]. These cases are performed at a pressure of $P=100 \mathrm{bar}$, which is above the critical pressures of oxygen and hydrogen $\left(P_{c}^{O_{2}}=50.4 b a r\right.$ and $\left.P_{c}^{H_{2}}=13.0 b a r\right)$. Transcritical conditions are reached by setting the oxygen stream temperature at $100 K$ (below its critical temperature: $T_{c}^{O_{2}}=154.6 \mathrm{~K}$ ), and the temperature of the hydrogen stream at $150 K$ (above $T_{c}^{H_{2}}=33.1 K$ ). Note that before comparing these approaches, it was verified that they result in the same perditions when no artificial dissipation is necessary for stabilization (Appendix B.1).

First, pure advection of density profiles were conducted to mimic the Euler convection of a parcel of oxygen into gas-like hydrogen. Second, a onedimensional Sod shock was used to investigate conservativity. Then, a twodimensional vortex case was performed, to replicate the effects of a turbulent eddy on a transcritical interface. And finally, a two-dimensional turbulent mixing layer was considered that reproduces the flow conditions at the exit of an a cryogenic rocket injector. 


\subsection{Advection test cases}

In the following, Euler convection of profiles of density, temperature and mass-fraction are examined. These profiles are advected across a one-dimensional domain (10 times the characteristic size of the profile, with inlet and outlet on both ends). Note that a periodic system is not considered here, in order to have an open system where the mean pressure is imposed by an outlet. This ensures an accuracte comparison of the three approaches. In a closed system (tested but not shown), the PB and HB approaches yield a constant mean pressure in time while the EB formulation predicts a decreasing system pressure as numerical dissipation acts as a numerical "evaporation". The profiles are described by a double hyperbolic tangent function that allows a precise control of the gradient initial magnitude and resolution. The initial profile is composed of oxygen at $100 \mathrm{~K}$ transitioning to gaseous hydrogen at $150 \mathrm{~K}$, at a pressure of 100bar. The transition thickness is set to $100 \mathrm{~nm}$, and the profile is convected at a velocity of $200 \mathrm{~m} / \mathrm{s}$ across a $5 \mathrm{~mm}$-long one-dimensional domaine. The domain is discretized with 200 uniformly distributed points. The schematic diagram of the computation is presented in Fig. 5. To exemplify the impact of numerical dissipation on the different approaches, a first-order accurate upwind scheme has been employed.

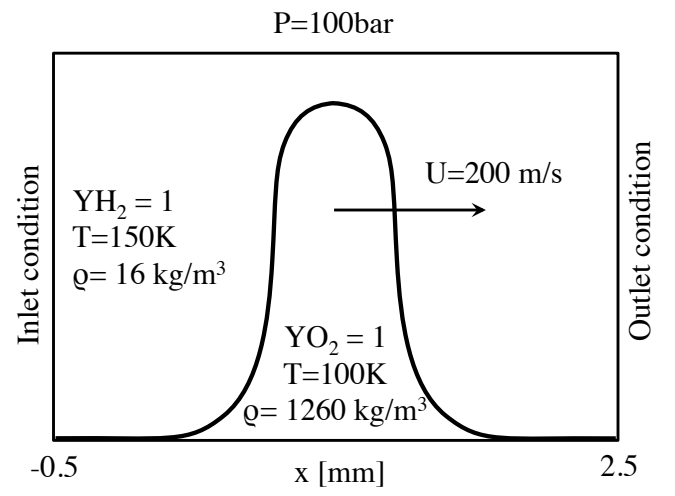

Figure 5: Schematics of the one-dimensional advection test. 
The spatial profiles of main flow quantities at $t=20 \mu \mathrm{s}$ are presented in Fig. 6. As discussed earlier in Section 2, the dissipation introduced by the up-

a)

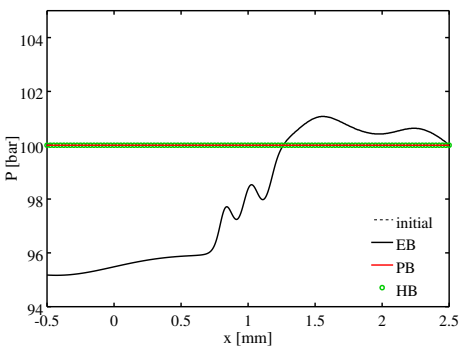

c)

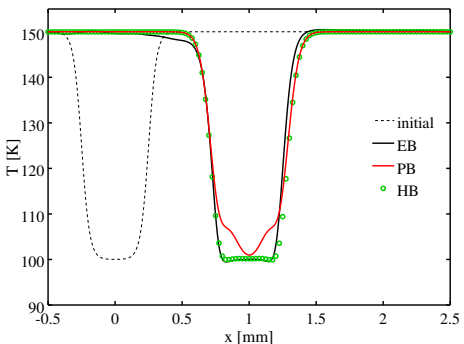

b)

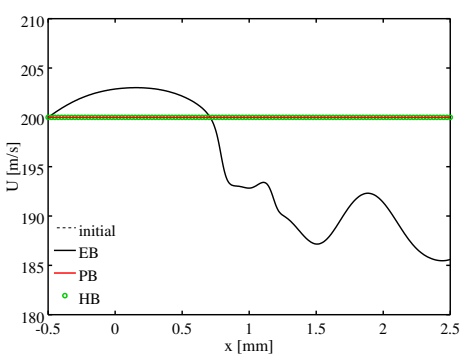

d)

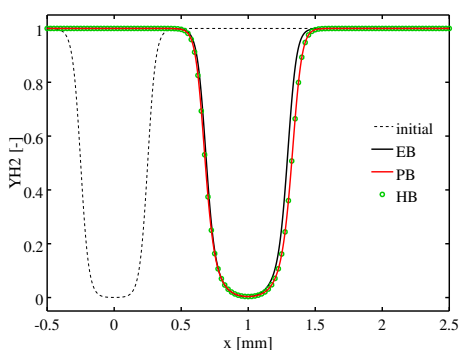

Figure 6: Spatial profiles of pressure (a), velocity (b), temperature (c) and hydrogen massfraction $(\mathrm{d})$, recorded at $t=20 \mu \mathrm{s}$ in the one-dimensional advection test (no molecular diffusion).

wind convective scheme significantly impacts the pressure and velocity fields of the energy-based approach, while being unaffected with the PB and HB methods. In the later methods, terms emanating from the stabilization scheme have been removed from the pressure equations preventing the spurious oscillations observed with the EB formulation. To compare the profiles in Fig. 6 (c), the temperature the energy-based approach is taken as reference since the methods is fully conservative. One can observed that the $\mathrm{PB}$ approach fails to recover the reference mixture temperature. This can be explained by the fact that density is dissipated while pressure remains constant. Maintaining pressure while dissipating density implies a local energy increase, reflected by a local increase in temperature as observed in Fig. 6 (c). Since no acoustic effects are present to redistribute energy, the error on temperature remains confined to the gra- 
dient region where the artificial dissipation of density occurs. This is similar to a high-pressure phase change where the pressure is artificially kept constant. Under transcritical conditions, if a dense parcel of fluid diffuses into a lighter fluid, the dense fluid extracts energy from its gas-like ambient (comparably to a low pressure evaporation) [16]. Under physical conditions, this process leads to small amplitude acoustic waves and velocity perturbations generated in the vicinity of the interface (permitting the redistribution of density). This is observed in the test case presented in Appendix B.1. However, if pressure is kept constant, energy is locally created by the thermodynamic scheme, (the system is non-conservative), and locally the temperature increases compared to an conservative approach. Matheis et al. [65] have observed a similar temperature behavior while computing the injection of a dense nitrogen jet into gaseous hydrogen at supercritical conditions. They noted a difference in the temperature fields predicted by a fully conservative approach (equivalent to the present EB method) and a pressure-based formulation. In the case of the energy-based approach, the temperature decreased below the injection temperature in the mixing layer between the dense nitrogen and the gaseous hydrogen. This was not observed when the pressure-based method was employed. No explanation was provided in [65] on the the cause of the discrepancy.

On the contrary, the HB approach provides a mixing temperature comparable to the energy-based method. Here, the thermodynamic scheme is based on the transported enthalpy which is almost identical to the one of the EB formulation. The small differences observed in Fig. 6 (c) are attributed to the local pressure variations present in the $\mathrm{EB}$ prediction.

Taking the energy-based results as reference, the differences on enthalpy and temperature can be explicitly formulated and measured. These errors read:

$$
\text { error } H_{P B}=\left(e_{\text {eos }}\left(\rho_{\text {trans }}, P_{\text {trans }}\right)+\frac{P_{\text {trans }}}{\rho_{\text {trans }}}-H_{E B}\right)
$$

and:

$$
\text { error } H_{H B}=\left(\frac{(\rho e)_{\text {trans }}+P_{\text {trans }}}{\rho_{\text {trans }}}-H_{E B}\right)
$$

The errors on $T$ are calculated by dividing the errors on enthalpy by $C_{p, \text { eos }}$. 
These departures in mass-fraction space are presented in Fig. 7. One can verify that the mixture enthalpy calculated in the HB approach agrees well with the one of the energy-based method. Small differences can be seen due to discrepancies in pressure fields. Significant errors are however measured for the pressure-based method since the energy recomputed from density and pressure is markedly different from the transported one. The same type of behavior is

a)

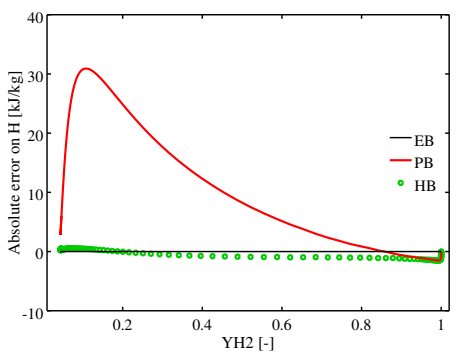

c)

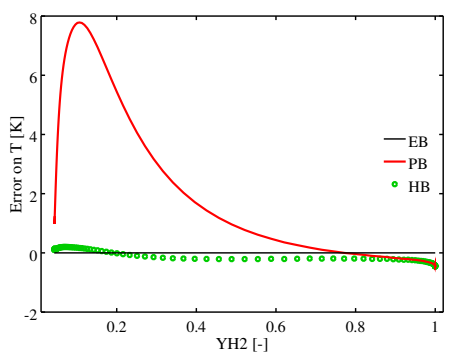

b)

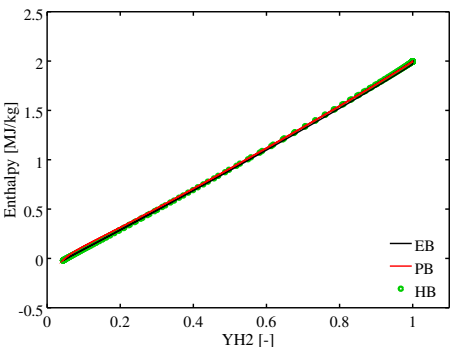

d)

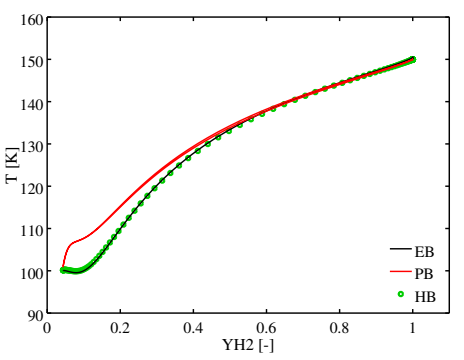

Figure 7: Measure in mass-fraction space of (a) error on enthalpy, (b) enthalpy, (c) error in temperature, and (d) temperature taking the EB approach as a reference. Profiles are based on the same dataset presented in Fig. 6, i.e. at $t=20 \mu \mathrm{s}$.

observed for all other thermodynamic quantities, a subset is presented in Fig. 8 . 
a)
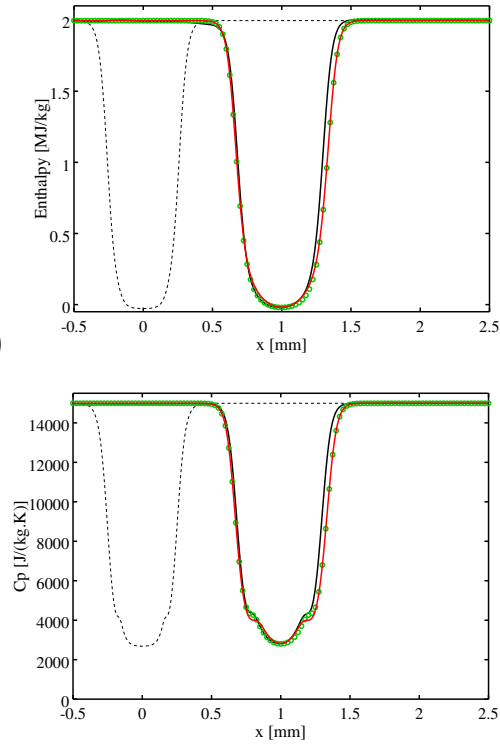

b)

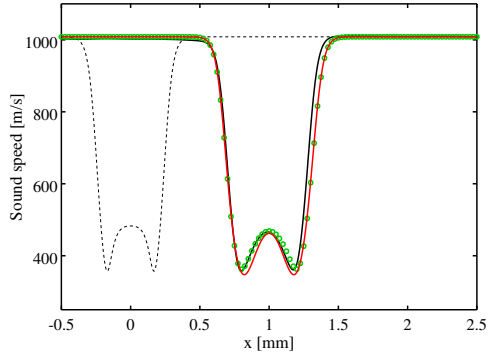

d)

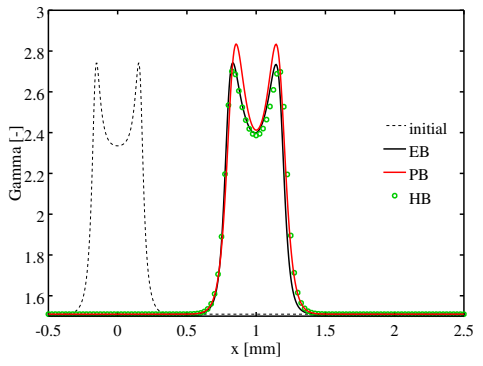

Figure 8: Key thermodynamic quantities estimated by the different methods in the onedimensional case: a) enthalpy in mixture space, b) speed of sound, c) heat capacity at constant pressure and d) heat capacity ratio. 


\subsection{Sod shock test}

The present Sod shock test [80] aims at distinguishing the different conservation properties of the three present approaches. The working fluid is pure oxygen. The left-hand-side state is defined by a pressure of 1 bar and a density of $1 \mathrm{~kg} / \mathrm{m}^{3}$, while the right-hand-side state is set at a pressure of $0.1 \mathrm{bar}$ and a density of $0.1 \mathrm{~kg} / \mathrm{m}^{3}$. The initial jump is characterized by a hyperbolic tangent profile with a thickness of $30 \mu \mathrm{m}$. The calculation is performed on a $2 \mathrm{~mm}$ long one-dimensional domain, discretized by 200 cells $\left(\Delta_{x}=10 \mu m\right)$. The Euler form of the transport system is used, and a first-order accurate upwind convective scheme is employed to ensure stability and enhance the impact of numerical dissipation.

Instantaneous profiles at $t=1 \mathrm{~ms}$ are presented in Fig. 9, and close-ups on the shock region are provided in the sub-figures. Focusing first on the similarities, all three methods predict the same locations for the expansion fan, and for the contact discontinuity. Because all methods rely on a first-order upwind oscillations are present in the results of the PB and HB approaches, since the pressure equation has been written without flux terms, and all derivatives are based on a second-order centered operator.

More differences can be observed in the shock region. One can observe that the energy-based approach predicts correctly the shock position and all thermodynamic quantities agree well with the analytical solution. As expected, the pressure-based approach fails to recover the correct shock speed since the approach is not conservative. Figure $9 f$ shows that the sensible energy downstream of the shock does not agree with the analytical solution. Subsequently, all thermodynamic quantities such as temperature, sound speed, and heat capacity present discrepancies compared to the reference. Finally, since the energy is transported in the HB approach, the sensible energy is correctly predicted downstream of the shock, which leads to better predictions of all thermodynamic quantities such as temperature and sound speed. This results in a more 
of sound), but does not exactly recover the shock speed. The main reason is that despite conserving energy, the HB method does not conserve enthalpy since the pressure equation is not used in its conservative form.

a)

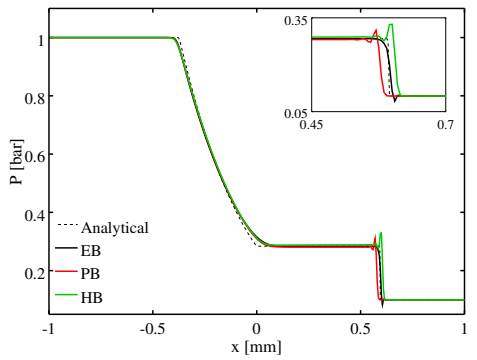

c)

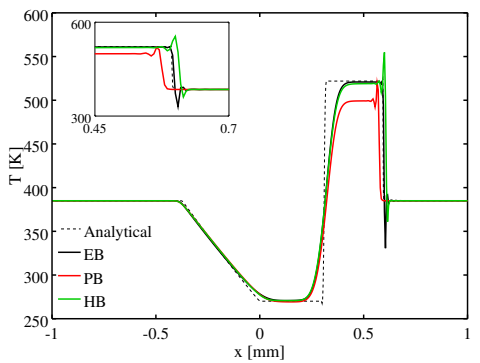

e)

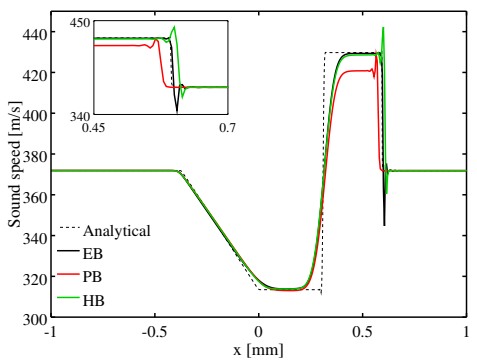

b)

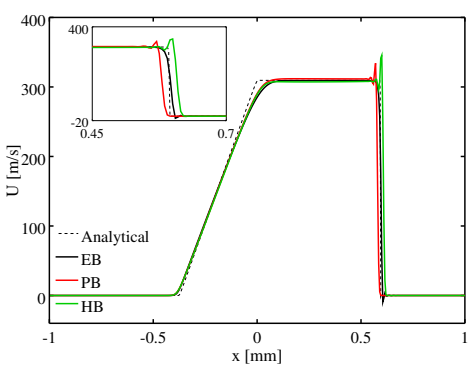

d)

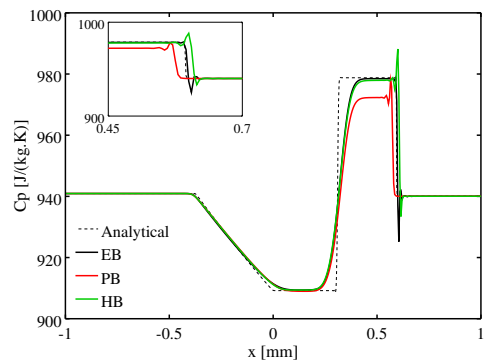

f)

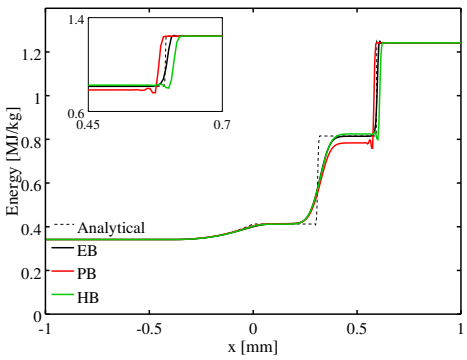

Figure 9: Sod shock in pure oxygen with a pressure ratio of ten-to-one: the sub-figure is a zoom on the shock region. Profiles captured at $t=1 \mathrm{~ms}$.

\subsection{Two-dimensional vortex test cases}

The objective of this section is to extend the previous comparisons to a more practical case where scalar gradients are resolved on only a few grid points and where the flow dynamics accentuates the resolution limitation. These aspects can be considered in a two-dimensional test case where a vortex interacts with a 
transcritical gradient. A density gradient is imposed between hydrogen at $150 \mathrm{~K}$ and oxygen at $100 K$ at $100 b a r$ using a hyperbolic tangent profile. Five points are now used to described the density jump. A $1 \mathrm{~mm} \times 1 \mathrm{~mm}$ two-dimensional domain is discretized by 200 points in each direction. A non-isentropic vortex is superimposed on the gradient to induce its deformation and compression. Note that in other vortex analysis (such as flame-vortex interaction) eddies are usually convected towards an interface to force their distortion. At present conditions, tests have shown that it was difficult to achieve sufficient strain by convecting a vortex towards the interface as the large density gradient acts as a quasi-solid wall to the gaseous vortical structure. This is why it was chosen to directly position the vortex across the density gradient. The circulation of the vortex is selected to generate enough deformation and strain to reach the resolution limit and activate the stabilization method. As described in Section 3.4, a flux limiter is employed to continuously transition from a second-order accurate QUICK scheme in zones where the numerical solution is sufficiently smooth to a first-order accurate upwind scheme in the gradient regions. No molecular diffusion is considered to isolate the impact of the stabilization approach.

The velocity field is described by [81]:

$$
\begin{aligned}
U & =-\frac{y C_{v}}{R_{v}^{2}} \exp \left(-\frac{x^{2}+y^{2}}{2 R_{v}^{2}}\right), \\
V & =\frac{x C_{v}}{R_{v}^{2}} \exp \left(-\frac{x^{2}+y^{2}}{2 R_{v}^{2}}\right)
\end{aligned}
$$

where $R_{v}=0.2 \mathrm{~mm}$ is the radius of the vortex and $C_{v}=-2.010^{-5} \mathrm{~m}^{2} / \mathrm{s}$ is the vortex circulation. A schematics of the computational domain and initial conditions are shown in Fig. 10.

Figure 11 compares the fields of pressure, flux limiter, and temperature given by the three methods at $t=2 \mu s$. The vortex creates a strong deformation of the interface along with a significant compression of the density gradient. In the compression zone, the gradient increases due to high strain-rate, which leads to a local increase of the flux limiter magnitude to stabilize the calculation (Fig. 11 475 b). Note that its magnitude is comparable between the three approaches. As 


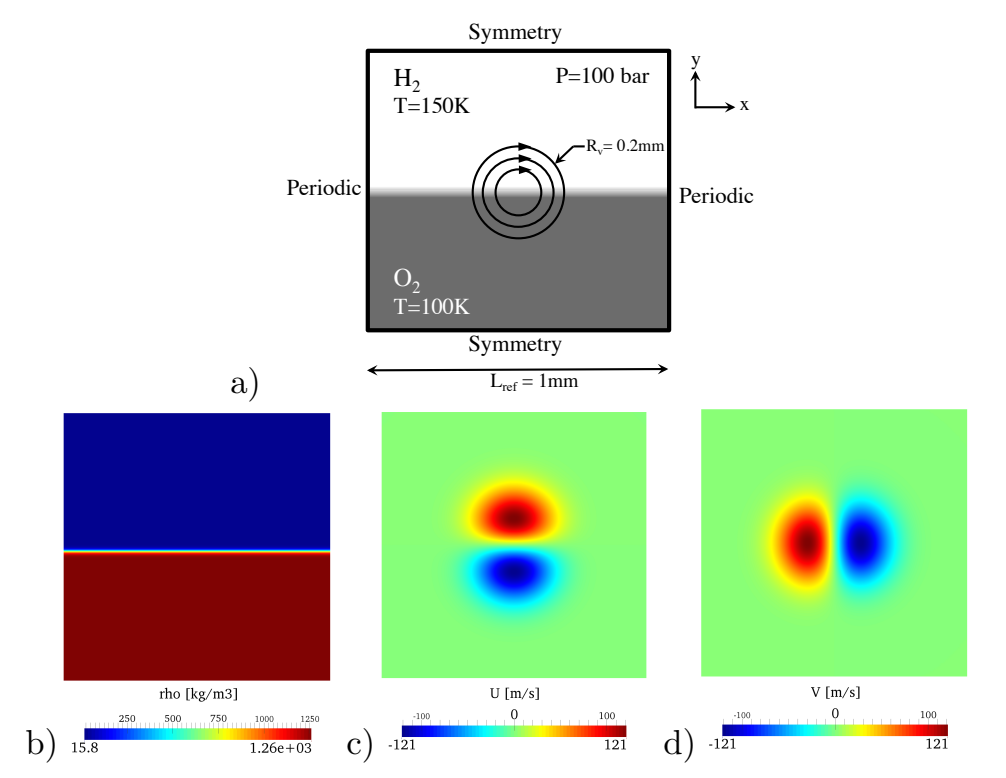

Figure 10: Schematics and initial conditions of the vortex-density gradient test case.

numerical dissipation is introduced, the energy-based approach is subject to pressure oscillations generated in the gradient region, while the pressure- and enthalpy-based methods exhibit smoother pressure fields (Fig. 11 a). The hydrodynamic effects of the vortex on the pressure field is however captured by the three approaches since they are related to spatial variation of momentum. A stability test shows that if the circulation of the vortex is increased, local pressure oscillations in the EB case result in very low pressure values, which locally drive the thermodynamic conditions inside the vapor dome. At these conditions, the dense fluid becomes a "classical" liquid, the continuum assumption is no longer valid and the equation of state becomes ill-conditioned. This leads to the divergence of the EB simulation while the PB and HB calculations remain stable.

The temperature field also shows some differences between the three formulations. Figures 11 (c) and (d) show that the energy-based form presents more pronounced temperature overshoots (in the $Y_{\mathrm{H}_{2}} \approx 1$ region of Fig. $11 \mathrm{c}$ ). The three approaches also behave differently in mass-fraction space. Figure 11 (d) 
shows that the pressure-based approach exhibits a local temperature increase $\left(Y_{H_{2}} \approx 0.05\right)$, contrary to the EB and HB formulations. This behavior is similar to what was previously observed in the advection test case in Section 4.1. In this figure, the adiabatic mixing line has been added. Adiabatic mixing is based on the assumption that the enthalpy varies linearly with mass-fraction, $h_{(T, Y)}=h_{\left(T_{O_{2}}, Y_{O_{2}}\right)}+Y_{H_{2}}\left(h_{\left(T_{H_{2}}, Y_{H_{2}}\right)}-h_{\left(T_{O_{2}}, Y_{O_{2}}\right)}\right)$. By construction, the adiabatic mixing line shows the thermodynamic state of the mixture if energy and species diffuse at the same rate, which is equivalent to assuming unity Lewis numbers for the considered species. For both the energy- and enthalpy-based approaches, the mixture temperature follows the adiabatic line since numerical dissipation impacts energy and density in a similar way. This is not the case for the PB method as only density is dissipated by the stabilization scheme. The reader is reminded that no physical diffusion is used for this test case (Euler simulation). Note also that numerical dissipation has not yet reached steady state, and this is why many mixture points are still away from the adiabatic mixing line in Fig. 11 (d). More scatter is also observed for the energy-based case due to compressibility effects and the presence of spurious pressure fluctuations. 
EB

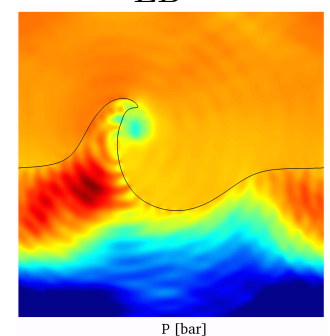

a)

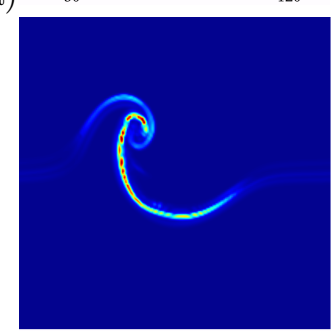

limiter [-]

b)

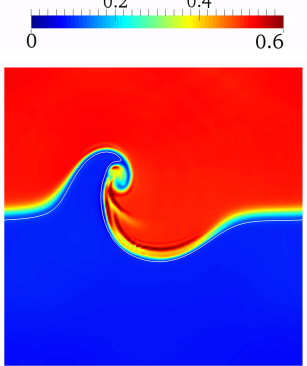

c)

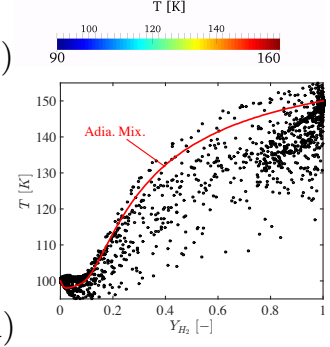

PB

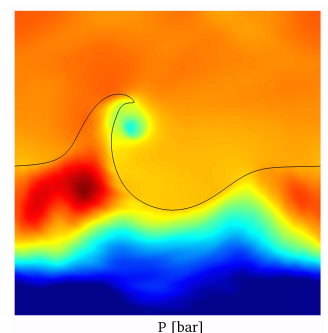

P [bar]
80
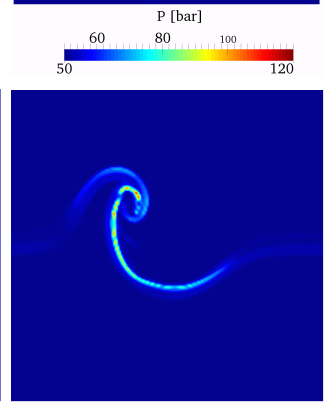

limiter [-]
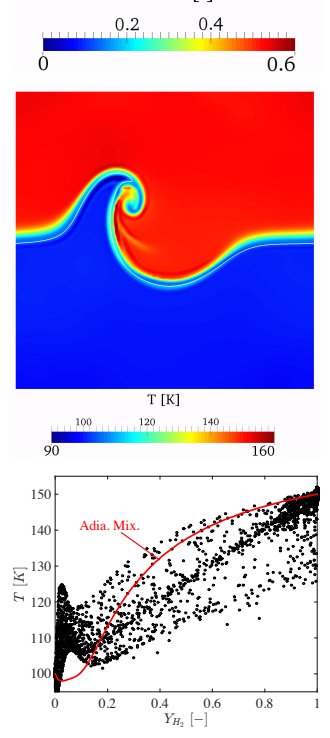

HB
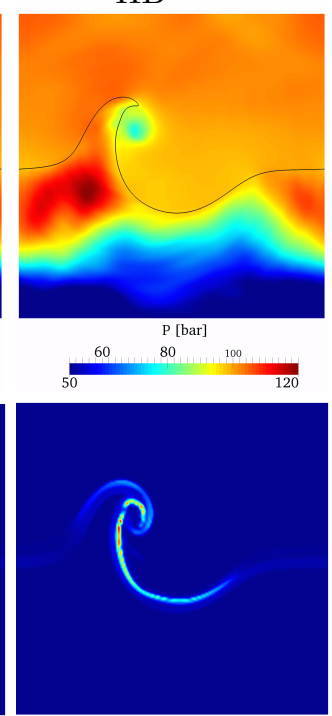

limiter [-]
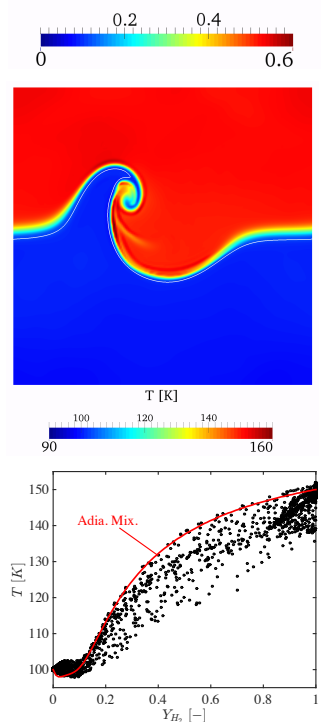

Figure 11: Vortex - density gradient interaction: impact of the EB, PB and HB methods on the instantaneous $(t=2 \mu s)$ a) pressure, b) flux limiter (limiter $=0:$ QUICK scheme, limiter $=1$ : 1st order upwind scheme) and c) temperature fields, with scatter plots of temperature versus mass-fraction of hydrogen presented in d). In figures a) and c), the line is the $\rho=500 \mathrm{~kg} / \mathrm{m}^{3}$ isoline. 


\subsection{Turbulent mixing layer} the early destabilization of the mixing layer forming between a dense stream of oxygen and a fast gas-like hydrogen stream at 100bar. This type of mixing layer occurs in rocket engines downstream of coaxial injectors where efficient mixing is obtained by the high shear generated between two injected streams. and detailed quantitative data are available for comparison. Note that for this final case molecular diffusion is active and all aspects previously investigated are present in the flow (i.e., diffusion, pure advection of dense elements, and turbulence-density gradient interactions).

520 ied by Ruiz et al. [79]. Their objective was to analyze the early destabilization of a LOX-GH2 mixing layer at rocket-like conditions using a near DNS grid resolution, and to provide a database for code comparison and validation. The dataset collected Ruiz et al. [79] contains results from a conservative and nonity fluctuations are imposed on inlet planes and velocity profiles follow a $1 / 7^{\text {th }}$ power law. The splitter is represented by an adiabatic wall condition. Pressure is imposed at the outlet. A sponge layer is employed downstream of $x=20 h$ to eliminate pressure oscillations potentially generated at the outlet. Top and

As strain rate reaches high values in the mixing layer, gradients are locally significant and numerical stabilization is required. As in the previous case, stabilization is provided by the numerical approach described in Section 3.4, 
where a quasi non-dissipative QUICK scheme is used in smooth flow regions and a dissipative first-order accurate upwind scheme is applied in gradients. In the present study, the grid is coarser than in [79]: in the mixing zone $(-3<y / h<3)$, the grid spacing is uniform $\left(\Delta_{x} / h=\Delta_{y} / h=1 / 100\right)$ against $\Delta_{x} / h=1 / 250$ in [79]. Outside the region of interest the grid is stretched at a rate of $0.1 \%$. A total of 3.7 million cells was employed for this configuration.

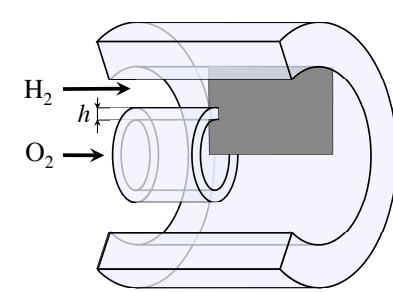

a)

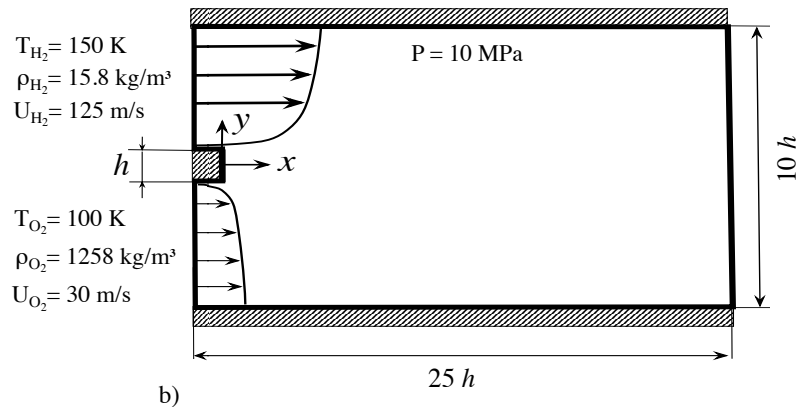

b)

$\stackrel{5}{5}$
Figure 12: Mixing layer configuration and computational domain. a) Typical coaxial injector of a liquid rocket engine (the grey frame represents the two-dimensional simulation domain). b) Computational domain dimensions and boundary conditions $(h=0.5 \mathrm{~mm})$.

In the following, instantaneous fields and temporal statistics (mean and rootmean square) are used to compare the three methods. Following recommendations from Ruiz and coworkers, a long period of fourteen flow-through time (based on the oxygen stream) was used to flush initialization transient and reach steady state. Time average statistics were then recorded for twenty flowthrough times. Statistics were sampled along one-dimensional lines across the computational domain at the same locations as in [79] (i.e., $x / h=1, x / h=3$, $x / h=5$ and $x / h=7)$.

Figure 13 presents the instantaneous flow fields of density and mass-fraction of hydrogen. Even if instantaneous fields appear different, the same dynamic 555 mechanisms were observed in the three methods. Those processes have previously been detailed by Ruiz et al. [79], thus only a brief summary is reported here.

Initial vortical structures are generated by a Kelvin-Helmoltz instability ini- 
tiated in the hydrogen stream downstream of the the backward-facing step. The in the mixing zone. Figure 17 shows the statistics of pressure. Mean pressure profiles compare closely between the three methods with a $0.1 \%$ shift for the PB approach compared to the EB and HB formalisms. Pressure diminishes slightly 


\section{$\mathrm{EB}$}

a)
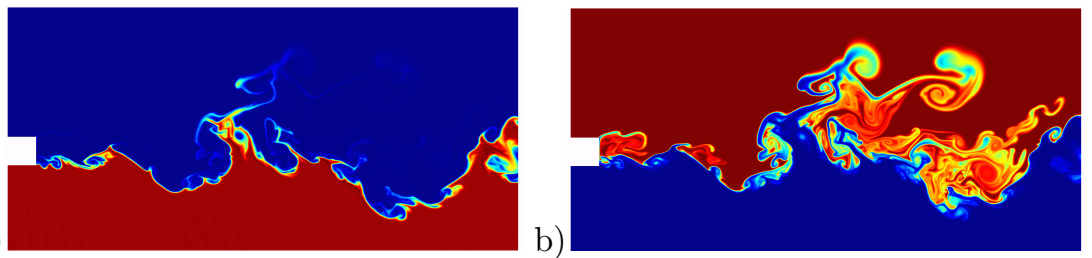

$\mathrm{PB}$
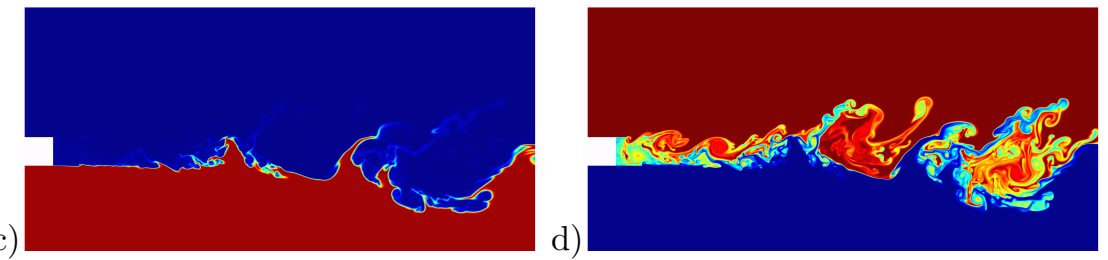

HB
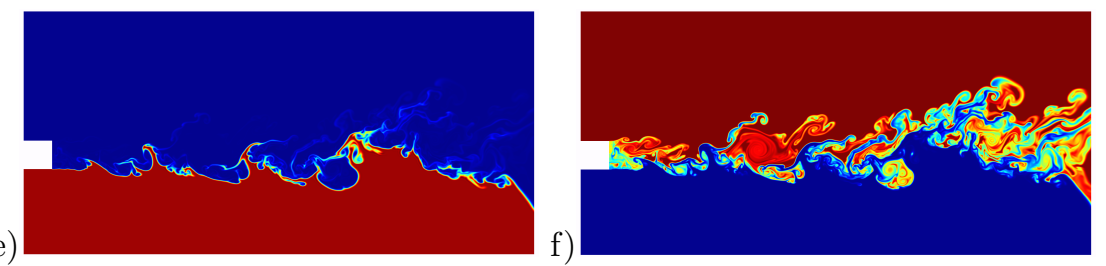

rho $[\mathrm{kg} / \mathrm{m} 3]$

YH2 [-]
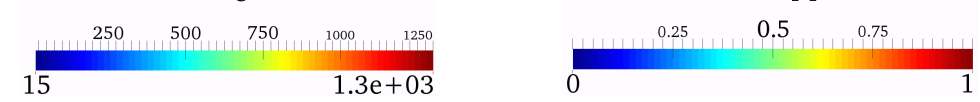

Figure 13: Comparison of density and hydrogen mass-fraction instantaneous fields computed with the three methods once flow dynamics reached steady state.

at the back of the splitter plate $(x / h=1)$ showing the foot-print of a recirculation zone in the wake of the splitter. Marked differences are however observed in the rms profiles presented in Fig. $17 \mathrm{~b}$ ), where large pressure variations are recorded in the energy-based approach, while only small amplitude variations are detected in both PB and HB cases. The large pressure perturbations recored in the EB case are the foot-print of a standing acoustic mode, triggered and fed by the spurious oscillations continuously generated in the gradient region. For sake of brevity the characterization of this sustained mode has not been included in the present work.

Finally, instantaneous and temporal statistics of temperature are analyzed. 

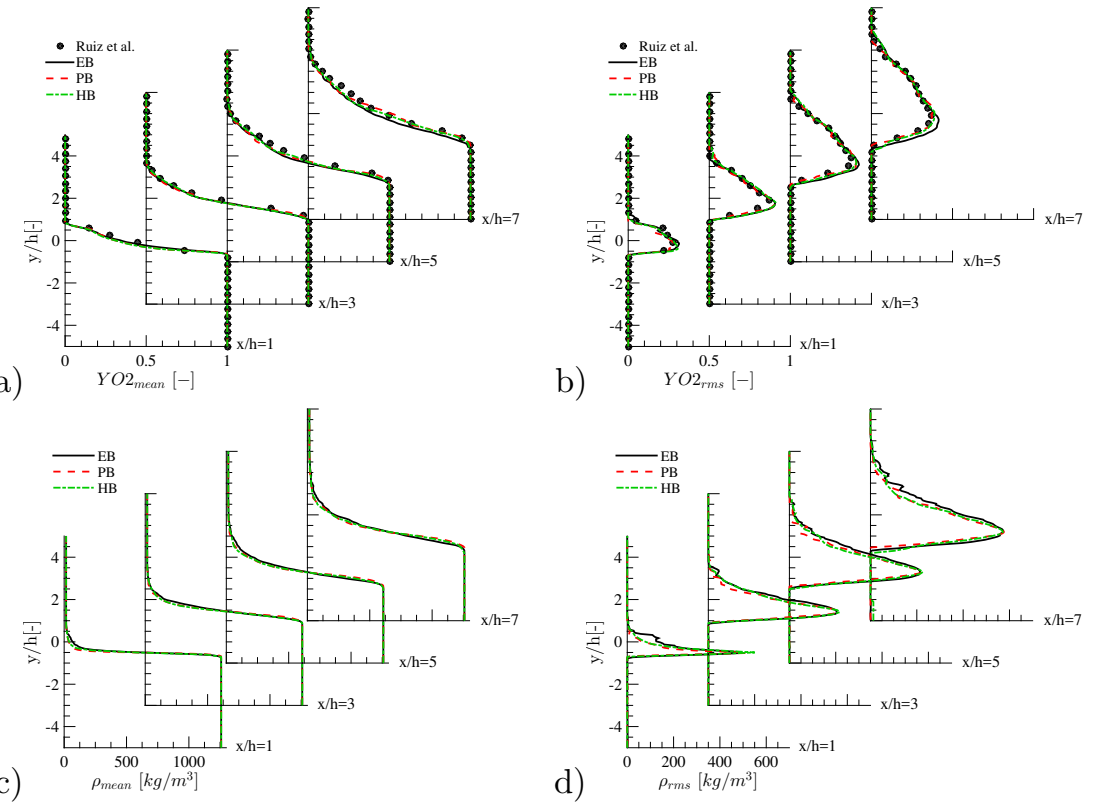

c)

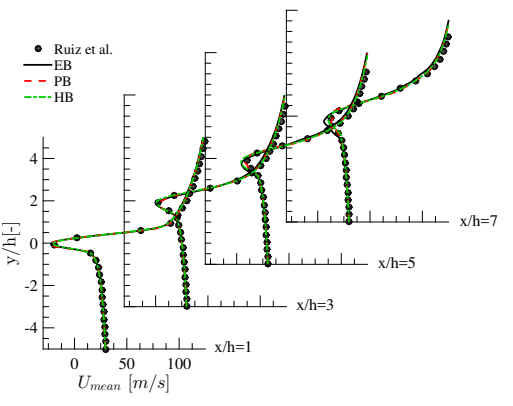

d)
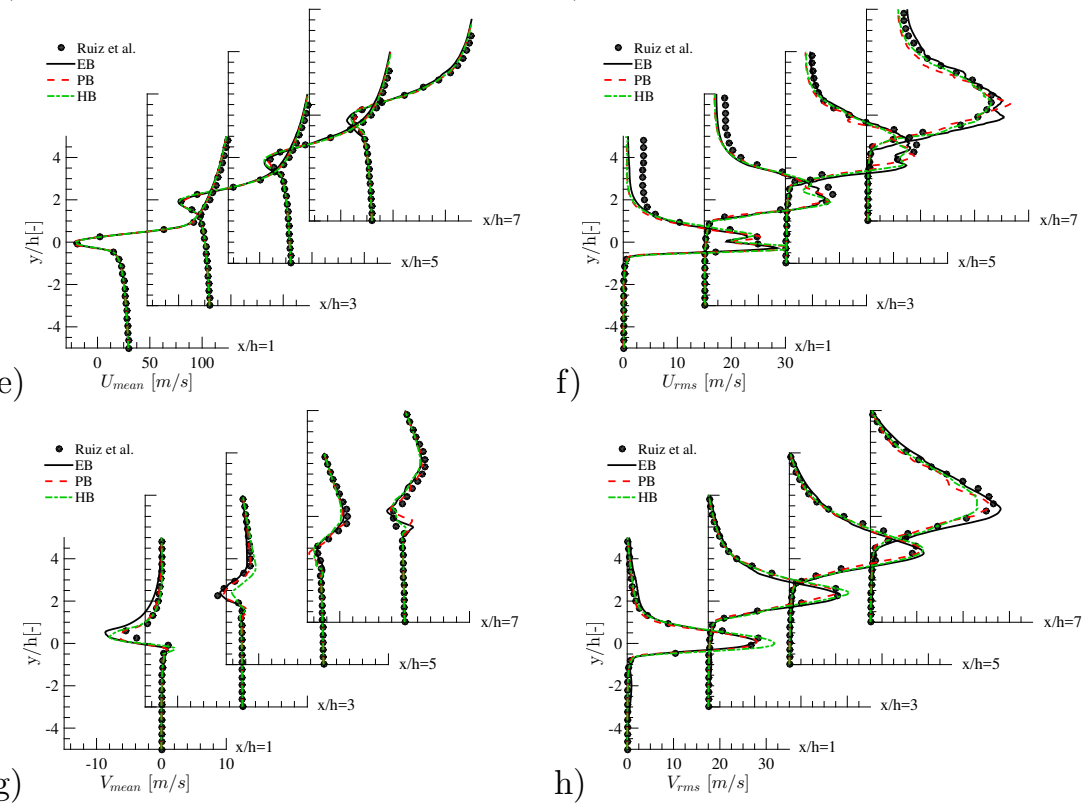

f)

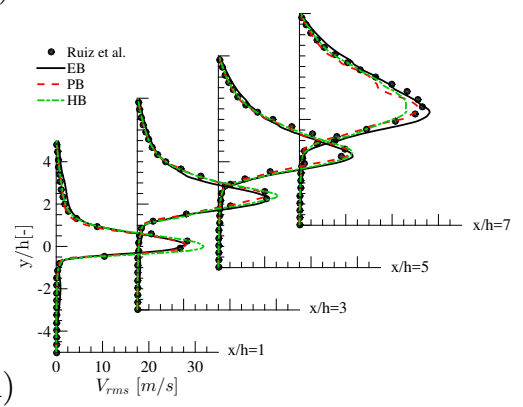

Figure 14: Statistical comparisons along one-dimensional transverse segments for oxygen massfraction,density, axial and transverse velocities. Symbols: database from Ruiz et al. [79]. Note: no reference data available for density. 
$\mathrm{EB}$

a)

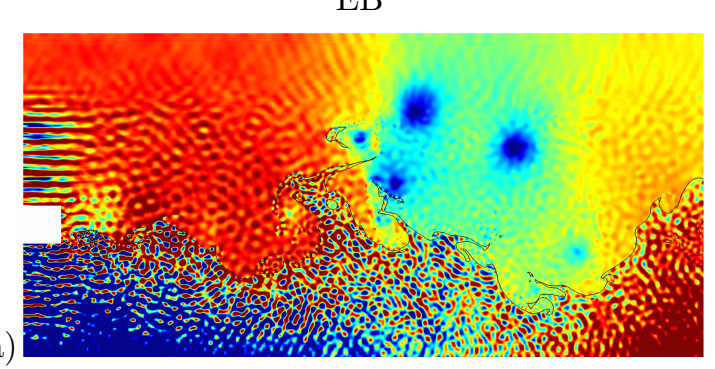

$\mathrm{PB}$

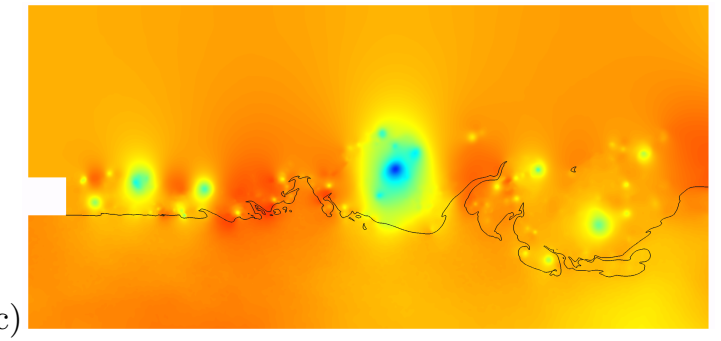

HB

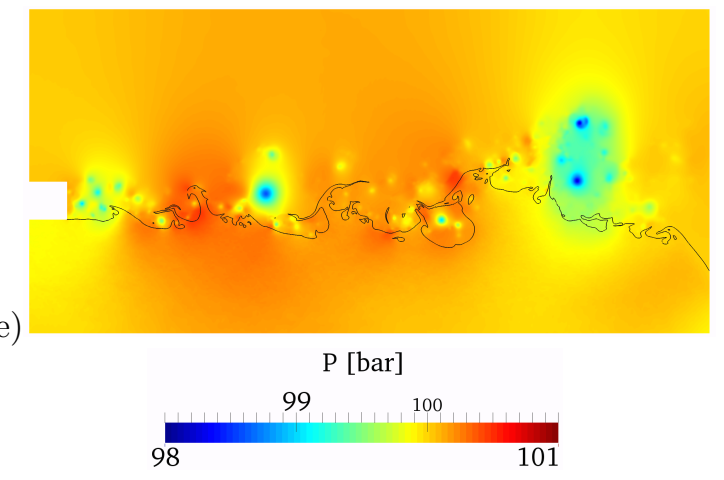

Figure 15: Comparison of instantaneous pressure fields between the three methods. The black iso-contour based on density $\left(\rho=600 \mathrm{~kg} / \mathrm{m}^{3}\right)$ localizes the density gradient.

The instantaneous temperature fields are shown in Fig. 18 along with the respective scatterplots in mass-fraction space. At first glance, all three methods result in very comparable temperature fields. However, the scatterplots indicate very different mixing behavior between the three approaches. It is worth noting that at current flow conditions, the Reynolds number is high $(R e=500,000)$ which make the molecular diffusion play a limited role in the mixing process. Subse- 


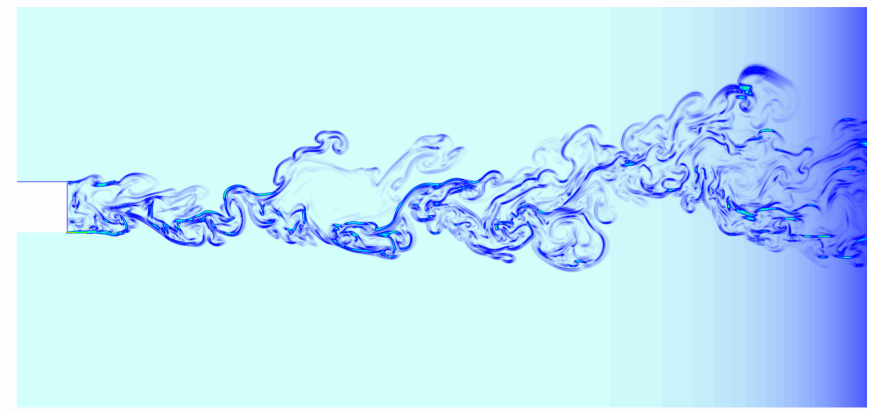

limiter [-]

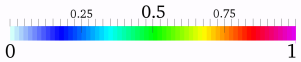

Figure 16: instantaneous field of limiter intensity used in the stabilization scheme. Only the EB case is reported here as all three methods are using the same limiter.

a)

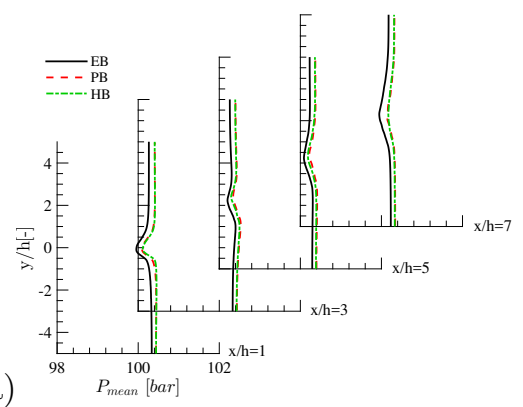

b)

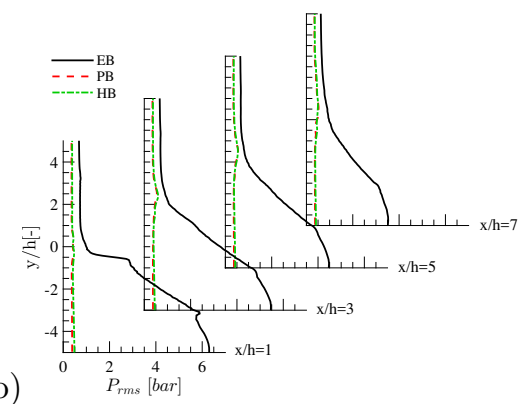

Figure 17: Comparisons of pressure mean and rms between the three approaches at different axial locations.

quent mixing depends predominantly on numerical dissipation as no turbulence closure is used for this study. This explains why in the EB and HB approaches, the temperature point clouds aggregate around the adiabatic mixing line, as previously observed in Section 4.3 In the PB method, however, mixture temperature is significantly increased in the gradient region since energy is created by the approach to dissipate density and mass-fractions without creating pressure fluctuation. Note that less scatter is observed with the HB approach than with the EB method. This is because higher levels of pressure fluctuations are observed in the energy-based case, which result in more local temperature fluc- 
tuations due to compressibility effects. These marked differences can also be

EB
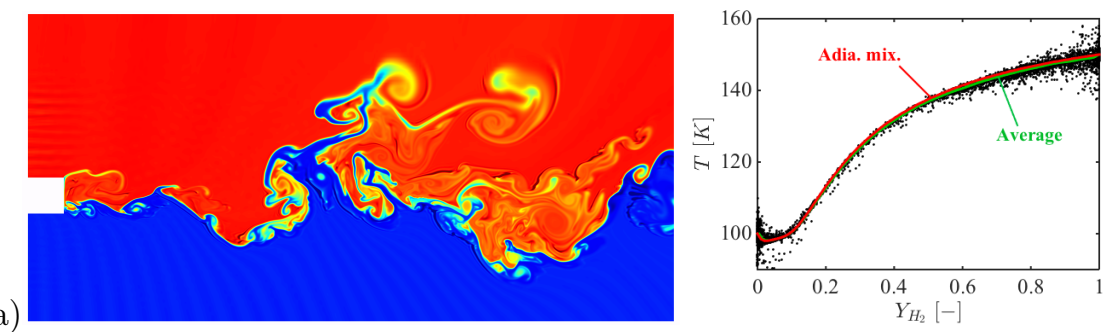

PB
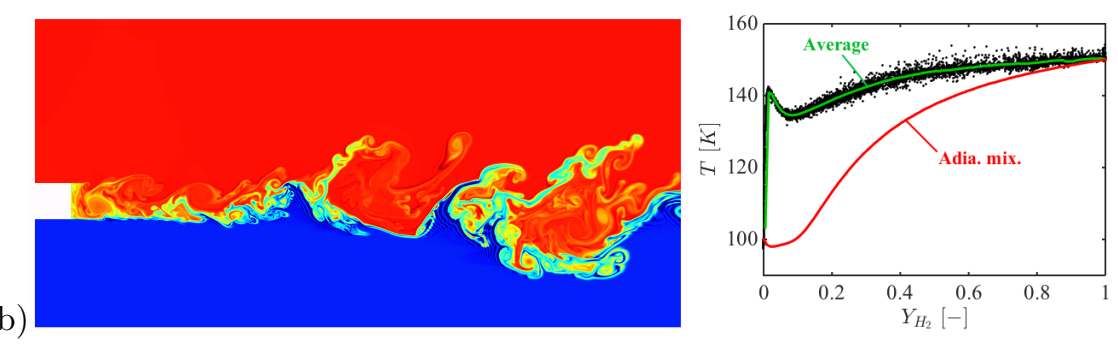

$\mathrm{HB}$
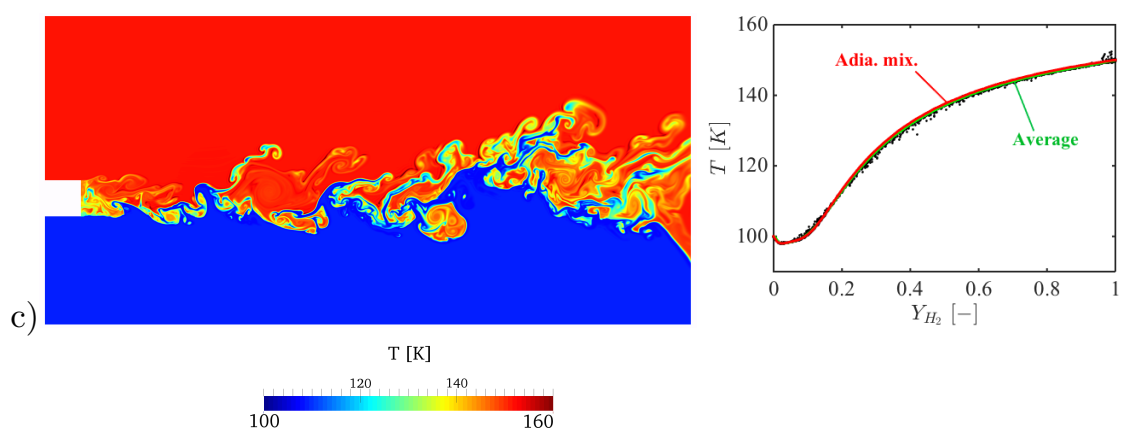

Figure 18: Comparison of instantaneous temperature fields obtained with the three different approaches. The associated scatterplots of temperature versus mass-fraction of hydrogen $\left(Y_{H_{2}}\right)$ are presented on the right-hand side.

615

observed in the temporal statistics presented in Fig. 19 showing mean and rms of temperature at different downstream locations. One can see that the PB method predicts higher mean temperature values in the mixing layer and more skewed $T_{r m s}$ profiles than the EB and HB approaches. The higher temperatures 620 in mass-fraction space observed in Fig. 18 b) for the pressure-based approach, 
result in a faster temperature transition between the two streams and a decrease in temperature intermittencies as less cold structures are generated. This leads to the skewed shape of $T_{r m s}$ for the PB method presented in Fig. $19 \mathrm{~b}$ ).

a)

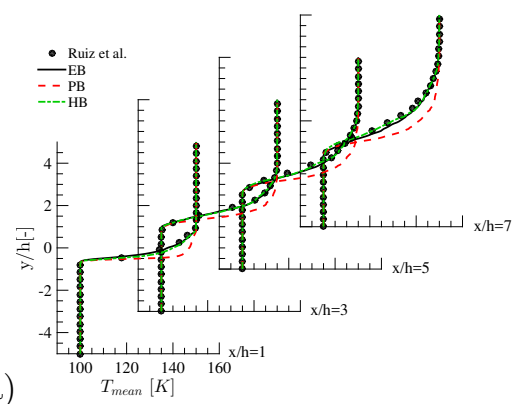

b)

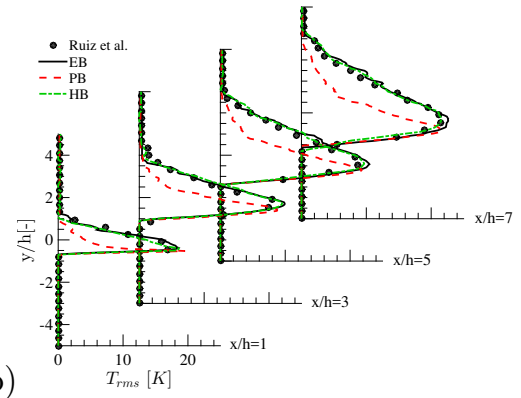

Figure 19: Comparisons of temperature mean and rms between the three approaches at different axial locations. Symbols: database from Ruiz et al. [79]

\section{Conclusions}

This investigation focuses on understanding the stability and mixing temperature issues encountered in transcritical flow simulations. Transcritical conditions are met when two fluids mix with thermodynamic states initially set on different sides of the pseudo-boiling line, and when the mean pressure is above the critical pressure of the mixture. At these conditions, thermodynamics exhibits a strong nonlinear behavior which translates to large Jacobian values in the mixing zone. This means that small variations in density and energy yield large pressure changes. This thermodynamic "sensitivity" significantly impacts the stability of flow solvers based on the conservative form of the Navier-Stokes equations (transport of density, total energy, momentum and species massfractions). In such solvers, small variations in transported quantities caused by the numerical approach (e.g., numerical dissipation or explicit filtering) lead to non-physical pressure fluctuations that modify the flow dynamics and may result in erroneous predictions or even the divergence of the computation.

Many approaches have been proposed to solve this stability issue. The 

the system of equations considered. The first approach (called energy-based) is a classical fully-conservative approach based on the transport of density, total energy, momentum and mass-fraction of species, and taken here as a reference. The second approach is a pressure-based method where the energy equation is tion of enthalpy is obtained by combining two transport equations on pressure and energy. In this manner, energy is transported, thus conserved, and spurious pressure oscillations can be controlled via the pressure equation. This method is however not fully conservative (enthalpy is not strictly conserved) since the pressure equation is not used in its conservative form: the residual terms from numerical dissipation have been intentionally removed from the equation to ensure dynamic stability.

These three methods have been implemented within the same numerical framework to facilitate comparison. A series of tests cases of increasing complexity has been considered to expose their different properties. Pure diffusion tests showed that all three methods behave identically. Pure advection tests revealed more marked differences. It was observed that if numerical dissipation was used, nonphysical pressure oscillations appeared in the predictions of the energy-based method. The pressure-based method remained dynamically stable since pressure is directly estimated from its transport equation which is free of numerical dissipation terms. However, its mixture temperature prediction significantly departed from the reference taken from the energy-based method. This is due to fact that density and mass-fractions are dissipated by the stabilization scheme while pressure remains constant. Thermodynamically this is only possible if energy is locally generated, causing the temperature to locally increase. The enthalpy-based formulation also provided oscillation-free flow fields, as well as a good agreement for the mixture temperature in comparison with the reference temperature from the fully-conservative approach. This improvement in thermodynamic representation is attributed to the fact that

the transported enthalpy and density are used as inputs to the thermodynamic 
scheme. This is not the case for the pressure-based method which uses transported pressure and density to reconstruct its thermodynamic state, with the pressure equation written in a nonconservative form. Conservation properties were also revealed in a shock test case where small errors in the shock positions were measured with the pressure- and enthalpy-based approaches. Deviations were mitigated in the HB predictions thanks to the better representation of the thermodynamics state.

Similar behaviors were observed in two more complex configurations, a vortex-interface case and a mixing layer, both at rocket-like conditions. Results revealed that in the case of the energy-based method, non-physical pressure oscillations were generated in regions where numerical dissipation is introduced by the stabilization scheme. In the mixing layer case, these artificial pressure fluctuations triggered and fed an acoustic oscillation which had significant impact on flow statistics, whereas pressure- and enthalpy-based predictions remained unaffected. A significant departure of the mixture temperature was however observed with the pressure-based approach, contrary to the enthalpybased method. In engineering applications, this erroneous thermodynamic state might impact predictions when slow chemical processes are key phenomena. This is the case in the estimation of the auto-ignition delay time of a diesel jet at elevated pressure, or the simulation of rocket pre-burner reacting flows under very rich or very lean mixture conditions.

\section{Appendix}

\section{Appendix A. Thermodynamics Jacobians}

This appendix is dedicated to deriving the Jacobian terms $(\partial P / \partial \rho)_{\rho e}$ and $(\partial P / \partial \rho e)_{\rho}$ in the case of a single species mixture.

Starting from the equations of Gibbs-Duhem [2] for density $\rho$ and energy $e$ for a single species system:

$$
d \rho=\left(\frac{\partial \rho}{\partial T}\right)_{P} d T+\left(\frac{\partial \rho}{\partial P}\right)_{T} d P
$$




$$
d e=\left(\frac{\partial e}{\partial T}\right)_{P} d T+\left(\frac{\partial e}{\partial P}\right)_{T} d P
$$

For notation simplification we introduce for $\phi=\rho$ or $e$ :

$$
\begin{aligned}
& \left(\frac{\partial \phi}{\partial T}\right)_{P, Y_{k}}=\phi_{T} \\
& \left(\frac{\partial \phi}{\partial P}\right)_{T, Y_{k}}=\phi_{P} \\
& \left(\frac{\partial \phi}{\partial Y_{k}}\right)_{T, P}=\phi_{k}
\end{aligned}
$$

We also define:

$$
\begin{aligned}
e & =h-\frac{P}{\rho} \\
d e & =C_{v} d T+\left[T\left(\frac{\partial P}{\partial T}\right)_{P}-P\right] d V \\
d h & =C_{p} d T+\left[V-T\left(\frac{\partial V}{\partial T}\right)_{P}\right] d P
\end{aligned}
$$

From previous relations, Eq. A.2 can be recast as:

$$
d e=\left(C_{p}+\frac{P}{\rho^{2}} \rho_{T}\right) d T+\frac{1}{\rho^{2}}\left(P \rho_{P}+T \rho_{T}\right) d P
$$

and by combining Eq. A.1 and A.9 one can obtain:

$$
d e=\left(\frac{\rho C_{p}}{\rho_{T}}+h\right) d \rho-\left(\frac{\rho C_{v} \rho_{P}}{\rho_{T}}\right) d P
$$

Using the relation: $C_{p}-C_{v}=T \rho_{T}^{2} /\left(\rho_{P} \rho^{2}\right)$, and developing $d \rho e=e d \rho+$ $\rho d e$, equation A.10 leads to the expression of the two Jacobians $(\partial P / \partial \rho)_{\rho e}$ and $700(\partial P / \partial \rho e)_{\rho}:$

$$
d P=\left(-\frac{\rho_{T}}{\rho C_{v} \rho_{P}}\right) d \rho e+\left(\frac{\rho C_{p}+h \rho_{T}}{\rho C_{v} \rho_{P}}\right) d \rho
$$

\section{Appendix B. Derivative relationships}

This appendix brings details necessary for the derivation of the pressurebased approach presented in Section 3.2. Here, the key derivatives used in the 
derivation are presented. Starting from the equation of Gibbs-Duhem [2] for

$$
\begin{gathered}
d \rho=\left(\frac{\partial \rho}{\partial T}\right)_{P, Y_{k}} d T+\left(\frac{\partial \rho}{\partial P}\right)_{T, Y_{k}} d P+\sum_{k=1}^{N}\left(\frac{\partial \rho}{\partial Y_{k}}\right)_{P, T} d Y_{k} \\
d e=\left(\frac{\partial e}{\partial T}\right)_{P, Y_{k}} d T+\left(\frac{\partial e}{\partial P}\right)_{T, Y_{k}} d P+\sum_{k=1}^{N}\left(\frac{\partial e}{\partial Y_{k}}\right)_{P, T} d Y_{k}
\end{gathered}
$$

By introducing enthalpy: $e=h-P / \rho$ and the heat capacities [2]:

$$
\begin{aligned}
d e & =C_{v} d T-\frac{1}{\rho^{2}}\left[T\left(\frac{\partial P}{\partial T}\right)_{\rho}-P\right] d \rho \\
d h & =C_{p} d T+\left[\frac{1}{\rho}+\frac{T}{\rho^{2}}\left(\frac{\partial \rho}{\partial T}\right)_{P}\right] d P,
\end{aligned}
$$

we obtain the following derivatives:

$$
\begin{aligned}
& e_{T}=C_{p}+\frac{P}{\rho^{2}} \rho_{T} \\
& e_{P}=-\frac{1}{\rho^{2}} \rho_{P}\left(T \frac{\partial P}{\partial T}-P\right)=\frac{1}{\rho^{2}}\left(P \rho_{P}+T \rho_{T}\right)
\end{aligned}
$$

Finally the Gibbs-Duhem system can be recast as:

$$
\begin{gathered}
d \rho=\rho_{T} d T+\rho_{P} d P+\sum_{k=1}^{N} \rho_{k} d Y_{k} \\
d e=\left[C_{p}+\frac{P}{\rho^{2}} \rho_{T}\right] d T+\frac{1}{\rho^{2}}\left[P \rho_{P}+T \rho_{T}\right] d P \\
+\sum_{k=1}^{N}\left[h_{k}+\frac{P}{\rho^{2}} \rho_{k}\right] d Y_{k}
\end{gathered}
$$

By combining B.7 and B.8 one can extract the variations of temperature $(d T)$ and pressure $(d P)$ with respect to those of density $(d \rho)$ energy $(d e)$ and species mass-fractions $\left(d Y_{k}\right)$ :

$$
d e=\left[\frac{T \rho_{T}}{\rho^{2}}-\frac{\rho_{P}}{\rho_{T}} C_{p}\right] d P+\left[\frac{C_{p}}{\rho_{T}}+\frac{P}{\rho^{2}}\right] d \rho+\sum_{k=1}^{N}\left[h_{k}-\frac{C_{p}}{\rho_{T}} \rho_{k}\right] d Y_{k}
$$


Using the relationship between the heat capacities [83]: $\frac{T \rho_{T}^{2}}{\rho^{2} \rho_{P}}=C_{p}-C_{v}$, equation B.9 can be recast as:

$$
d e=\left[-\frac{\rho_{P}}{\rho_{T}} C_{v}\right] d P+\left[\frac{C_{p}}{\rho_{T}}+\frac{P}{\rho^{2}}\right] d \rho+\sum_{k=1}^{N}\left[h_{k}-\frac{C_{p}}{\rho_{T}} \rho_{k}\right] d Y_{k}
$$

This leads to the variation of pressure:

$$
d P=\frac{C_{P} \rho^{2}+P \rho_{T}}{C_{v} \rho_{P} \rho^{2}} d \rho-\frac{\rho_{T}}{\rho_{P} C_{v}} d e+\sum_{k=1}^{N} \frac{\rho_{T}}{\rho_{P} C_{v}}\left[h_{k}-\frac{C_{p}}{\rho_{T}} \rho_{k}\right] d Y_{k}
$$

and by introducing the speed of sound: $c^{2}=C_{p} /\left(C_{v} \rho_{P}\right)$, the pressure variation reads:

$$
d P=c^{2}\left[1+\frac{P \rho_{T}}{C_{p} \rho^{2}}\right] d \rho-\frac{\rho_{T} c^{2}}{C_{p}} d e+\sum_{k=1}^{N} \frac{c^{2}}{C_{p}}\left[\rho_{T} h_{k}-C_{p} \rho_{k}\right] d Y_{k}
$$

A similar relationship can be derived for $d T$ by combining B.7 and B.8:

$$
d e=\frac{1}{\rho^{2}}\left[P+\frac{\rho^{2}}{\rho_{T}}\left(C_{p}-C_{v}\right)\right] d \rho+C_{v} d T+\sum_{k=1}^{N}\left[h_{k}-\frac{T \rho_{T} \rho_{k}}{\rho^{2} \rho_{P}}\right] d Y_{k}
$$

which, by introducing $\gamma=C_{p} / C_{v}$ leads to:

$$
d T=-\left[\frac{P}{\rho^{2} C_{v}}+\frac{\gamma-1}{\rho_{T}}\right] d \rho+\frac{1}{C_{v}} d e+\sum_{k=1}^{N}\left[\frac{T \rho_{T} \rho_{k}}{\rho^{2} \rho_{P} C_{v}}-\frac{h_{k}}{C_{v}}\right] d Y_{k}
$$

Appendix B.1. Pure-diffusion test case

The objective of this test case is to verify that all three methods provide similar results for pure diffusion, when no artificial dissipation or filtering is employed under transcritical conditions. A one-dimensional gradient between pure oxygen at $100 \mathrm{~K}$ and gaseous hydrogen at $150 \mathrm{~K}$, is initialized at a pressure of 100bar. The physical domain is $2 \mu \mathrm{m}$ long and the transition zone about $0.5 \mu \mathrm{m}$ thick, described by 25 grid points. The domain is bounded by symmetry conditions. The schematic diagram of the computation is presented in Fig. B.20. 


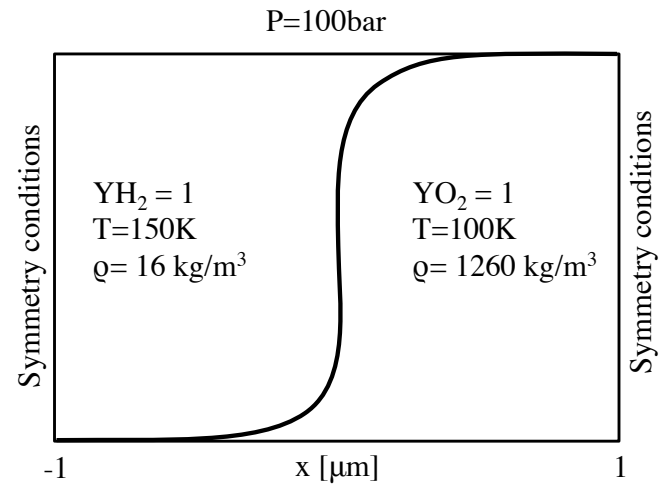

Figure B.20: Schematics of the one-dimensional diffusion test.

Figure B.21 shows the the evolution of keys quantities during the early diffusion transients predicted by the three methods. One can first observe that the three approaches are in good agreement with each other. Slight differences can be detected in the instantaneous pressure profiles. Pressure is evaluated by very different procedures between the three approaches, and the thermodynamics being very non-linear, these pressure differences are attributed to small numerical deviations. The drift in mean pressure can be explained by the relaxation of energy between the two sides of the gradient (Fig. B.21 (d)). The system being closed and adiabatic, the total energy is conserved as well as mass. An equilibrium analysis shows that at the present conditions, a system containing one percent of hydrogen in volume has an asymptotic pressure of $44 \mathrm{bar}$ and an asymptotic temperature of $98 \mathrm{~K}$. Present conditions also lead to a fast relaxation of the thermodynamic conditions, which results in significant instantaneous pressure and velocity variations (Fig. B.21 (e)).

In Figure B.21 (f), the adiabatic mixing line has been added. Adiabatic mixing is based on the assumption that the enthalpy varies linearly with massfraction, $h_{(T, Y)}=h_{\left(T_{O_{2}}, Y_{O_{2}}\right)}+Y_{H_{2}}\left(h_{\left(T_{H_{2}}, Y_{H_{2}}\right)}-h_{\left(T_{O_{2}}, Y_{O_{2}}\right)}\right)$. By construction, the adiabatic mixing line shows the thermodynamic state of the mixture if energy and species diffuse at the same rate, which is equivalent to assuming unity Lewis numbers for the considered species. By adding this line to Fig. B.21 (f), one can 
see that diffusion mixing of the $\mathrm{O} 2-\mathrm{H} 2$ system is different from a unity Lewis number diffusion due to differential diffusion effects. In addition, the balance in sensible energy causes the temperature to locally (at $Y_{H_{2}} \approx 0.05$ in Fig. B.21 (f)) decrease below the initial minimum value. This test shows that the three approaches behave very similarly in the case of a pure diffusion process when no artificial dissipation is required for stabilization or explicit filtering is applied to the transported quantities.

a)

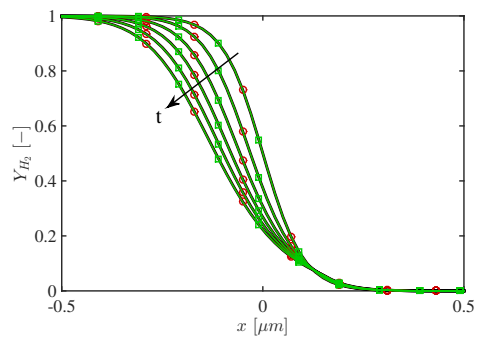

c)

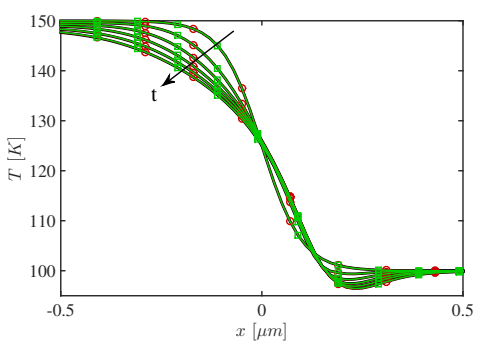

e)

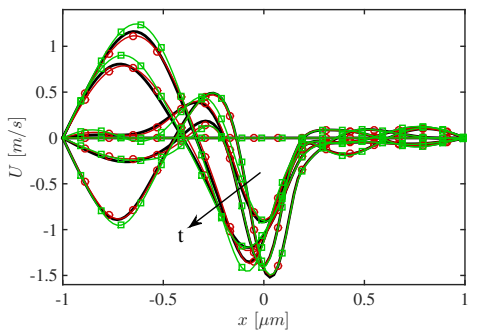

b)

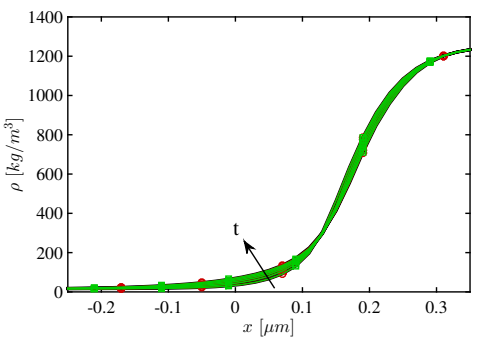

d)
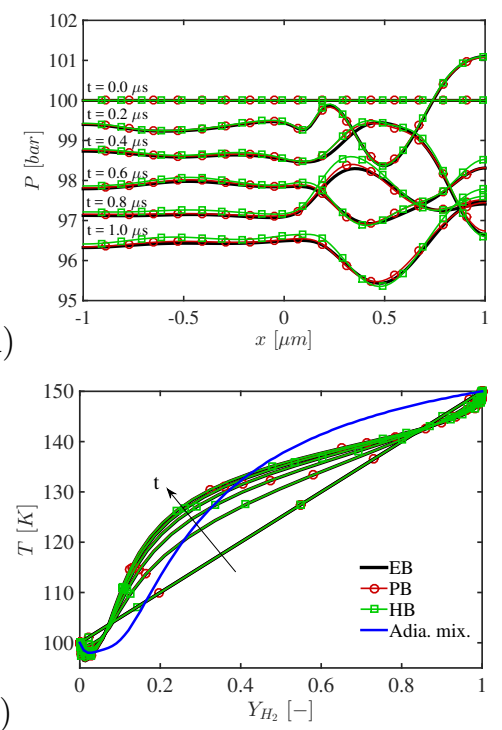

Figure B.21: 1D diffusion test: comparison between the EB (black lines), PB (red line with circles) and HB (green line with squares). In each figure, time is indicated by an arrow. Precise time marks are shown in Fig.B.21 (d). Note that Figs. (a), (b) and (c) have been zoomed in to get better details on the gradient region. 


\section{Appendix C. Stabilization approach}

The present stabilization method is based on the composition of a highorder scheme and a robust upwind scheme [78]. Historically, such methods were developed to ensure stable solution of supersonic flows [84, 85]. In simplified notations, the inviscid flux $\mathbf{F}_{C}$ on a given cell face can be expressed as: $\mathbf{F}_{C}=\mathbf{F}_{C}^{\text {high }}+\nu\left(\mathbf{F}_{C}^{\text {low }}-\mathbf{F}_{C}^{\text {high }}\right)$, where $\mathbf{F}_{C}^{\text {high }}$ and $\mathbf{F}_{C}^{\text {low }}$ are the contributions of the high-order and low-order schemes, respectively. $\nu$ is a sensor defined on the interval $0 \leq \nu \leq 1$. This term switches the truncation error from high-order accuracy when $\nu \rightarrow 0$, in smooth regions of the flow, to low-order accuracy when $\nu \rightarrow 1$ in shocks and steep gradients. In the present study, $\mathbf{F}_{C}^{h i g h}$ is the second-order accurate QUICK scheme (Quadratic Upstream Interpolation for Convective Kinematics) [77] and $\mathbf{F}_{C}^{\text {low }}$ a first-order upwind scheme.

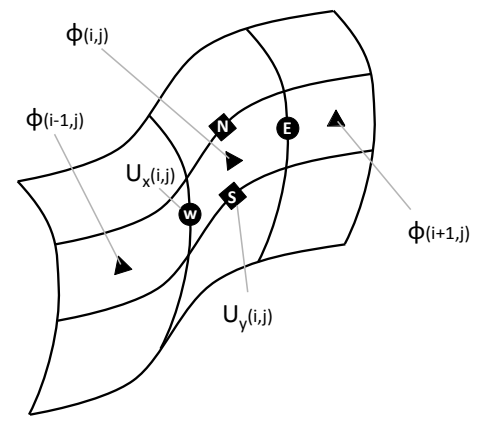

Figure C.22: Data storage in the present staggered formulation in a simplified two-dimensional example. $\phi$ is the vector of transported scalars, $U_{x}$ and $U_{y}$ are the velocities in the x- and y-curvilinear directions, respectively.

Practically, in the present solver, the convective flux (per unit area) for any convected quantity $\phi$ is evaluated using the following upwind approach:

$$
\begin{aligned}
& \mathbf{F}_{C, \phi}^{W}=U_{x}^{W} \phi^{L}, \text { if } U_{x}^{W}>0, \\
& \mathbf{F}_{C, \phi}^{W}=U_{x}^{W} \phi^{R}, \text { if } U_{x}^{W}<0 .
\end{aligned}
$$

The formulation for momentum terms is similar and thus not described here. Note, however, that cross-terms for momentum fluxes require an additional interpolation step. 
The flux limiter is incorporated in the definition of $\phi^{L}$ and $\phi^{R}$, defined here in an one-dimensional context:

$$
\begin{aligned}
\phi^{L} & =\frac{1}{2}\left(\phi_{i}+\phi_{i-1}\right)-\frac{1-\nu_{i-1 / 2}(\phi)}{8}\left(\phi_{i}-2 \phi_{i-1}+\phi_{i-2}\right)-\frac{\nu_{i-1 / 2}(\phi)}{2}\left(\phi_{i}-\phi_{i-1}\right), \\
\phi^{R} & =\frac{1}{2}\left(\phi_{i}+\phi_{i-1}\right)-\frac{1-\nu_{i-1 / 2}(\phi)}{8}\left(\phi_{i+1}-2 \phi_{i}+\phi_{i-1}\right)-\frac{\nu_{i-1 / 2}(\phi)}{2}\left(\phi_{i}-\phi_{i-1}\right) .
\end{aligned}
$$

One can observe that when $\nu_{i-1 / 2}(\phi)=0$ (in smooth regions of the flow), the $Q U I C K$ formulation is recovered:

$$
\phi^{L}=\frac{1}{2}\left(\phi_{i}+\phi_{i-1}\right)-\frac{1}{8}\left(\phi_{i}-2 \phi_{i-1}+\phi_{i-2}\right)=\frac{3}{8} \phi_{i}+\frac{3}{4} \phi_{i-1}-\frac{1}{8} \phi_{i-2},
$$

and in gradient zones, $\nu_{i-1 / 2}(\phi)=1$, a stable first-order accurate upwind scheme is used:

$$
\phi^{L}=\frac{1}{2}\left(\phi_{i}+\phi_{i-1}\right)-\frac{1}{2}\left(\phi_{i}-\phi_{i-1}\right)=\phi_{i-1}
$$

In practice, switch values are estimated at cell centroids and then interpolated at cell faces:

$$
\nu_{i-1 / 2}(\phi)=\min \left(1, \max \left(0, \frac{\nu_{i}(\phi)+\nu_{i-1}(\phi)}{2}\right)\right)
$$

In the context of supercritical flows with large density gradients, the main challenge is to define an optimal switch $\nu$ that detects under-resolved region of the flow. Jorgenson and Turkel have compared the different existing switching approaches and have proposed a modified formulation of the van-Leer and vanAlbada switches that present the best properties for hyperbolic problems [86]. Lacaze et al. [87] recently proposed a new sensor maintaining better symmetry properties. This switch is used in the present study.

\section{Appendix D. Residual terms of the stabilization scheme in the pres- sure equation}

In the $\mathrm{PB}$ and $\mathrm{HB}$ approaches, pressure oscillations are eliminated by removing the terms resulting from the artificial dissipation (from the stabilization 
method) applied to the other conserved quantities ( $\rho, \rho e, \rho \mathbf{U}$, and $\left.\rho Y_{k}\right)$ in the pressure equation. The stabilization strategy used in the present work relies on upwinding the convective fluxes in gradient regions (see description in Appendix C). For a given transported quantity $\phi$, the residual of the convective term can be decomposed into a centered term and a dissipation term. For example, in the case of a one-dimensional configuration with a left-to-right flow, a first order upwind residual (upwinded towards the left) would be written in delta form (for the cell of index $i$ ) as:

$$
R_{1 s t O}=\frac{d t}{V_{i}}\left(\phi_{i}(S U)_{i+1 / 2}-\phi_{i-1}(S U)_{i-1 / 2}\right),
$$

with $d t$ the time step, $V_{i}$ the volume of the cell of index $i, S_{i+1 / 2}$ the surface of the left cell face and $U_{i+1 / 2}$ the flow velocity normal to the left surface. This residual can be re-written as:

$$
R_{1 s t ~}=R_{\text {centered }}+R_{\text {dissip } \phi},
$$

with

$$
\begin{gathered}
R_{\text {centered }}=\frac{d t}{V_{i}}\left(\frac{\phi_{i+1}+\phi_{i}}{2}(S U)_{i+1 / 2}-\frac{\phi_{i}+\phi_{i-1}}{2}(S U)_{i-1 / 2}\right), \\
R_{\text {dissip } \phi}=-\frac{d t}{V_{i}}\left(\frac{\phi_{i+1}-\phi_{i}}{2}(S U)_{i+1 / 2}-\frac{\phi_{i}-\phi_{i-1}}{2}(S U)_{i-1 / 2}\right) .
\end{gathered}
$$

where $R_{\text {dissip } \phi}$ is the dissipation residual of the quantity $\phi$ produced by the 1st order accurate upwind scheme. When these terms are re-introduced in the pressure equation, the pressure-based recovers the behavior of the energybased approach and dynamic stability is disrupted by the apparition of spurious pressure oscillations. These pressure source terms can be explicitly formulated as:

$$
\begin{aligned}
d P_{\text {dissip } \rho} & =\left(\frac{\partial P}{\partial \rho}\right)_{\rho e, \rho \mathbf{U}, \rho Y_{k}} R_{\text {dissip } \rho} \\
d P_{\text {dissip } \rho e} & =\left(\frac{\partial P}{\partial \rho e}\right)_{\rho, \rho \mathbf{U}, \rho Y_{k}} R_{\text {dissip } \rho e} \\
d P_{\text {dissip } \rho U_{i}} & =\left(\frac{\partial P}{\partial \rho U_{i}}\right)_{\rho, \rho e, \rho U_{j} j \neq i, \rho Y_{k}} R_{\text {dissip } \rho U_{i}} \\
d P_{\text {dissip } \rho Y_{k}} & =\left(\frac{\partial P}{\partial \rho Y_{k}}\right)_{\rho, \rho e, \rho \mathbf{U}, \rho Y_{l} l \neq k} R_{\text {dissip } \rho Y_{k}}
\end{aligned}
$$


where:

$$
\begin{array}{rlr}
\left(\frac{\partial P}{\partial \rho}\right)_{\rho e, \rho \mathbf{U}, \rho Y_{k}} & =\frac{c^{2}}{\rho C_{p}}\left[C_{p}\left(\rho+\sum_{k=1}^{N_{s}-1} Y_{k} \rho_{Y_{k}}\right)+\rho_{T}\left(h_{t}-\mathbf{U}^{2}-\sum_{k=1}^{N_{s}-1} Y_{k} \mathcal{H}(\not D)\right) \rho\right) \\
\left(\frac{\partial P}{\partial \rho e}\right)_{\rho, \rho \mathbf{U}, \rho Y_{k}} & =-\frac{\rho_{T} c^{2}}{\rho C_{p}} & \text { (D.10) } \\
\left(\frac{\partial P}{\partial \rho U_{i}}\right)_{\rho, \rho e, \rho U_{j} j \neq i, \rho Y_{k}} & =U_{i} \frac{\rho_{T} c^{2}}{\rho C_{p}} & \text { (D.11) } \\
\left(\frac{\partial P}{\partial \rho Y_{k}}\right)_{\rho, \rho e, \rho \mathbf{U}, \rho Y_{l} l \neq k} & =\frac{c^{2}}{\rho C_{p}}\left(\rho_{T} \mathcal{H}_{k}-\rho_{Y_{k}} C_{p}\right) & \text { (D.12) }
\end{array}
$$

In both the pressure-based and enthalpy-based approaches, these terms are intentionally removed from the pressure equation to avoid spurious oscillations. 


\section{Acknowledgments}

Support for this research was provided by the U. S. Department of Energy (DOE), Office of Energy Efficiency and Renewable Energy (EERE), Vehicle Technologies (VT) program, under grant number VT0401000. Support was also

provided by the U.S. DOE Office of Basic Energy Sciences (BES) Division of Chemical Sciences, Geosciences, and Biosciences, under contract DE-AC04-94AL85000. Sandia National Laboratories is operated and managed by National Technology and Engineering Solutions of Sandia, LLC., a wholly owned subsidiary of Honeywell International, Inc.

\section{References}

[1] J. Hirschfelder, F. Curtiss, R. Bird, Molecular theory of gases and liquids, John Wiley \& Sons, New York, 1964.

[2] R. C. Reid, J. M. Prausnitz, B. E. Polling, The properties of liquids and gases, Vol. fourth ed., McGraw Hill, New York, 1987.

[3] M. Oschwald, J. J. Smith, R. Branam, J. Hussong, A. Schik, B. Chehroudi, D. Talley, Injection of fluids into supercritical environments, Combustion science and technology 178 (1-3) (2006) 49-100.

[4] R. N. Dahms, J. C. Oefelein, On the transition between two-phase and single-phase interface dynamics in multicomponent fluids at supercritical pressures, Physics of Fluids 25 (092103) (2013) 1-24. doi:10.1063/1. 4820346.

[5] R. D. Woodward, D. G. Talley, Raman imaging of transcritical cryogenic propellants, AIAA paper 468 (1996) 1996.

[6] J. Hickey, P. Ma, M. Ihme, S. Thakur, Large eddy simulation of shear coaxial rocket injector: Real fluid effects, in: 49th AIAA/ASME/SAE/ASEE Joint Propulsion Conference, 2013, pp. 4071-4085. 
[7] H. Terashima, M. Koshi, Approach for simulating gas-liquid-like flows under supercritical pressures using a high-order central differencing scheme, Journal of Computational Physics 231 (20) (2012) 6907-6923.

[8] S. Kawai, H. Terashima, H. Negishi, A robust and accurate numerical method for transcritical turbulent flows at supercritical pressure with an arbitrary equation of state, Journal of Computational Physics 300 (2015) $116-135$.

[9] T. Schmitt, L. Selle, A. Ruiz, B. Cuenot, Large-eddy simulation of supercritical-pressure round jets, AIAA journal 48 (9) (2010) 2133-2144.

[10] L. Cutrone, M. Ihme, M. Herrmann, Modeling of high-pressure mixing and combustion in liquid rocket injectors, in: Proceedings of the Summer Program, Center for Turbulence Research, NASA AMES, Stanford University, USA, 2006, pp. 269-282.

825 [11] S. Pohl, M. Jarczyk, M. Pfitzner, B. Rogg, Real gas CFD simulations of hydrogen/oxygen supercritical combustion, in: Progress in Propulsion Physics, Vol. 4, EDP Sciences, 2013, pp. 583-614.

[12] F. Demoulin, S. Zurbach, A. Mura, High-pressure supercritical turbulent cryogenic injection and combustion: A single-phase flow modeling proposal, Journal of Propulsion and Power 25 (2) (2009) 452-464.

[13] M. Poschner, M. Pfitzner, CFD-Simulation of the Injection and Combustion of LOX and H2 at Supercritical Pressures, American Institute of Aeronautics and Astronautics, 2010, pp. 1144-1156. doi:10.2514/6.2010-1144.

[14] L. Qiu, R. D. Reitz, Simulation of supercritical fuel injection with condensation, International Journal of Heat and Mass Transfer 79 (2014) 1070-1086.

[15] D. T. Banuti, K. Hannemann, Supercritical Pseudo-Boiling and its Relevance for Transcritical Injection, American Institute of Aeronautics and Astronautics, 2014, pp. 2014-3571. doi:10.2514/6.2014-3571. 
[16] D. Banuti, K. Hannemann, The absence of a dense potential core in supercritical injection: A thermal break-up mechanism, Physics of Fluids 28 (3) (2016) 035103.

[17] D. Banuti, V. Hannemann, K. Hannemann, B. Weigand, An efficient multifluid-mixing model for real gas reacting flows in liquid propellant rocket engines, Combustion and Flame 168 (2016) 98-112.

[18] J. Bellan, Supercritical (and subcritical) fluid behavior and modeling : drops, streams, shear and mixing layers, jets and sprays, Progress in Energy and Combustion Science 26 (2000) 329-366, eR.

[19] R. Miller, K. Harstad, J. Bellan, Direct numerical simulations of supercritical fluid mixing layers applied to heptane-nitrogen, Journal of Fluid Mechanics 436 (6) (2001) 1-39.

[20] N. Okong'o, J. Bellan, Consistent boundary conditions for multicompoment real gas mixtures based on characteristic waves, Journal of Computational Physics 176 (2002) 330-344.

[21] N. Okong'o, J. Bellan, Consistent large-eddy simulation of a temporal mixing layer laden with evaporating drops. part 1. direct numerical simulation, formulation and a priori analysis, Journal of Fluid Mechanics 499 (2004) $1-47$.

[22] J. Bellan, Theory, modeling and analysis of turbulent supercritical mixing, Combustion science and technology 178 (1-3) (2006) 253-281.

[23] E. Masi, J. Bellan, K. G. Harstad, N. A. Okong'o, Multi-species turbulent mixing under supercritical-pressure conditions: modelling, direct numerical simulation and analysis revealing species spinodal decomposition, Journal of Fluid Mechanics 721 (2013) 578-626.

[24] J. Foster, R. S. Miller, A priori analysis of subgrid mass diffusion vectors in high pressure turbulent hydrogen/oxygen reacting shear layer flames, Physics of Fluids 24 (7) (2012) 075114. 
[25] C. B. da Silva, G. Balarac, O. Metais, Transition in high velocity ratio coaxial jets analysed from direct numerical simulations, Journal of Turbulence 4 (24) (2003) 1-18.

[26] H. Tani, S. Teramoto, N. Yamanishi, K. Okamoto, A numerical study on a temporal mixing layer under transcritical conditions, Computers \& Fluids 85 (2013) 93-104.

[27] J. C. Oefelein, V. Yang, Modeling High-Pressure Mixing and Combustion Processes in Liquid Rocket Engines, Journal of Propulsion and Power14 (5) (1998) 843-857.

[28] N. Zong, G. Ribert, V. Yang, A flamelet approach for modeling of liquid oxygen (lox)/methane at supercritical pressures, in: Forty-sixth AIAA aerospace sciences meeting and exhibit, AIAA, Vol. 946, 2008, pp. 1-16.

[29] X. Petit, G. Ribert, G. Lartigue, P. Domingo, Large-eddy simulation of supercritical fluid injection, The Journal of Supercritical Fluids 84 (2013) 61-73.

[30] G. Lacaze, A. Misdariis, A. Ruiz, J. C. Oefelein, Analysis of high-pressure diesel fuel injection processes using LES with real-fluid thermodynamics and transport, Proceeding of the Combustion Institute 35 (2015) 16031611. doi:10.1016/j.proci.2014.06.072.

[31] N. Tramecourt, M. Masquelet, S. Menon, Large-eddy simulation of unsteady wall heat transfer in a high pressure combustion chamber, in: 41 st AIAA/ASME/SAE/ASEE Joint Propulsion Conference \& Exhibit, 2005, pp. 1-17.

[32] X. Petit, G. Ribert, P. Domingo, Lox/ch4 mixing and combustion under supercritical conditions, in: 51st AIAA Aerospace Sciences Meeting including the New Horizons Forum and Aerospace Exposition, 2013, pp. 713-723. 
[33] N. Tramecourt, S. Menon, J. Amaya, LES of supercritical combustion in a gas turbine engine, in: 40th AIAA/ASME/SAE/ASEE Joint Propulsion Conference and Exhibit, 2004, pp. 3381-3392.

[34] S. Matsuyama, J. Shinjo, S. Ogawa, Y. Mizobuchi, Large eddy simulation of lox/gh2 shear-coaxial jet flame at supercritical pressure, in: 48th AIAA Aerospace Sciences Meeting Including the New Horizons Forum and Aerospace Exposition, 2010, pp. 208-218.

[35] S. Matsuyama, Correlation of optical emission and turbulent length scale in a coaxial jet diffusion flame, Combustion and Flame 161 (4) (2014) 937949.

[36] J. C. Oefelein, Thermophysical characteristics of shear-coaxial LOX-H2 flames at supercritical pressure, Proceedings of the Combustion Institute 30 (2) (2005) 2929-2937.

[37] J. C. Oefelein, LES of Supercritical LOX-H2 Injection and Combustion in a Shear-Coaxial Uni-Element Rocket, American Institute of Aeronautics and Astronautics, 2003, pp. 1-13. doi:10.2514/6.2003-479.

[38] J. C. Oefelein, Mixing and combustion of cryogenic oxygen-hydrogen shearcoaxial jet flames at supercritical pressure, Combustion Science and Technology 178 (1-3) (2006) 229-252.

[39] S. Matsuyama, J. Shinjo, Y. Mizobuchi, S. Ogawa, A numerical investigation on shear coaxial lox/gh2 jet flame at supercritical pressure, in: 44th AIAA Aerospace Sciences Meeting and Exhibit, 2006, pp. 761-772.

[40] R. Smith, G. Xia, W. A. Anderson, C. L. Merkle, Computational simulations of the effect of backstep height on nonpremixed combustion instability, AIAA Journal 48 (9) (2010) 1857-1868. doi:10.2514/1.40385.

[41] T. S. Park, LES and RANS simulations of cryogenic liquid nitrogen jets, The Journal of Supercritical Fluids 72 (2012) 232 - 247. doi:http://dx. doi.org/10.1016/j.supflu. 2012.09.004. 
[42] H. Müller, M. Pfitzner, J. Matheis, S. Hickel, Large-eddy simulation of coaxial LN2/GH2 injection at trans-and supercritical conditions, Journal of Propulsion and Power 32 (1) (2015) 46-56.

[43] J. Matheis, H. Müller, C. Lenz, M. Pfitzner, S. Hickel, Volume translation methods for real-gas computational fluid dynamics simulations, The Journal of Supercritical Fluids 107 (2016) 422-432.

[44] H. Meng, V. Yang, A unified treatment of general fluid thermodynamics and its application to a preconditioning scheme, Journal of Computational Physics 189 (1) (2003) 277-304. doi:10.1016/S0021-9991(03)00211-0.

[45] M. Masquelet, S. Menon, Y. Jin, R. Friedrich, Simulation of unsteady combustion in a LOX-GH2 fueled rocket engine, Aerospace Science and Technology 13 (8) (2009) 466-474.

[46] X. Wang, V. Yang, Supercritical mixing and combustion of liquidoxygen/kerosene bi-swirl injectors, Journal of Propulsion and Power 33 (2) (2016) 316-322.

[47] H. Huo, V. Yang, Large-eddy simulation of supercritical combustion: Model validation against gaseous h2-o2 injector, Journal of Propulsion and Power 33 (5) (2017) 1272-1284. doi:10.2514/1.B36368.

URL https://doi.org/10.2514/1.B36368

[48] H. Terashima, S. Kawai, N. Yamanishi, High-resolution numerical method for supercritical flows with large density variations, AIAA journal 49 (12) (2011) 2658-2672.

[49] N. Zong, H. Meng, S. Hsieh, V. Yang, A numerical study of cryogenic fluid injection and mixing under supercritical conditions, Physics of Fluids 16 (2004) 4248-4261.

[50] N. Zong, V. Yang, A Numerical study of high-pressure oxygen/methane mixing and combustion of a shear coaxial injector, in: 43rd AIAA Aerospace Sciences Meeting and Exhibit, Vol. 152, 2005, pp. 1-15. 
[51] N. Zong, V. Yang, Cryogenic fluid jets and mixing layers in transcritical and supercritical environments, Combustion Science and Technology 178 (1-3) (2006) 193-227.

[52] N. Zong, V. Yang, Near-field flow and flame dynamics of lox/methane shear-coaxial injector under supercritical conditions, Proceedings of the Combustion Institute 31 (2) (2007) 2309-2317.

[53] T. Schmitt, Y. Méry, M. Boileau, S. Candel, Large-eddy simulation of oxygen/methane flames under transcritical conditions, Proceedings of the Combustion Institute 33 (1) (2011) 1383-1390.

[54] T. Schmitt, J. Rodriguez, I. Leyva, S. Candel, Experiments and numerical simulation of mixing under supercritical conditions, Physics of Fluids 24 (5) (2012) 055104-055104.

[55] L. Selle, T. Schmitt, Large-eddy simulation of single-species flows under supercritical thermodynamic conditions, Combustion Science and Technology 182 (4-6) (2010) 392-404.

[56] L. Hakim, A. Ruiz, T. Schmitt, M. Boileau, G. Staffelbach, S. Ducruix, B. Cuenot, S. Candel, Large eddy simulations of multiple transcritical coaxial flames submitted to a high-frequency transverse acoustic modulation, Proceedings of the Combustion Institute 35 (2) (2015) 1461-1468.

[57] A. Urbano, L. Selle, G. Staffelbach, B. Cuenot, T. Schmitt, S. Ducruix, S. Candel, Exploration of combustion instability triggering using large eddy simulation of a multiple injector liquid rocket engine, Combustion and Flame 169 (2016) 129-140.

[58] P. Ma, L. Bravo, M. Ihme, Supercritical and transcritical real-fluid mixing in diesel engine applications, in: Proceeding of the Summer Program 2014, CTR Stanford, 2014, pp. 99-108. 
[66] C. Pantano, R. Saurel, T. Schmitt, An oscillation free shock-capturing method for compressible van der Waals supercritical fluid flows, Journal of Computational Physics 335 (2017) 780-811.

[67] D. Y. Peng, D. B. Robinson, A new two-constant equation of state, Indus-

[59] E. Rinaldi, R. Pecnik, P. Colonna, Exact jacobians for implicit navierstokes simulations of equilibrium real gas flows, Journal of Computational Physics 270 (2014) 459-477.

[60] E. Taşkinoğlu, J. Bellan, Subgrid-scale models and large-eddy simulation of oxygen stream disintegration and mixing with a hydrogen or helium stream at supercritical pressure, Journal of Fluid Mechanics 1 (1) (2011) 1-38.

[61] L. Selle, N. Okong'o, J. Bellan, K. Harstad, Modelling of subgrid-scale phenomena in supercritical transitional mixing layers: an a priori study, Journal of Fluid Mechanics 593 (1) (2007) 57-91.

[62] S. Karni, Hybrid multifluid algorithms, SIAM Journal on Scientific Computing 17 (5) (1996) 1019-1039.

[63] H. Terashima, M. Koshi, Unique characteristics of cryogenic nitrogen jets under supercritical pressures, Journal of Propulsion and Power 29 (6) (2013) 1328-1336.

[64] M.-M. Jarczyk, M. Pfitzner, Large Eddy Simulation of Supercritical Nitrogen Jets, American Institute of Aeronautics and Astronautics, 2012, pp. 1-13. doi:10.2514/6.2012-1270.

[65] J. Matheis, H. Müller, M. Pfitzner, S. Hickel, Large Eddy Simulation of Cryogenic Coaxial LN2/GH2 Injection Under Supercritical Pressures, in: International Symposium on Turbulence and Shear Flow Phenomena (TSFP-9), Melbourne, Australia, 2015, pp. 1-6.

trial \& Engineering Chemistry Fundamentals 15 (1) (1976) 59-64. 
[68] P. Linstrom, W. Mallard, NIST Chemistry WebBook, NIST Standard Reference database, Vol. 69, National Institute of Standards and Technology (NIST), Gaithersburg MD, 20899, 2005.

URL http://webbook.nist.gov

[69] S. Gordon, B. J. McBride, Computer program for calculation of complex chemical equilibrium compositions, rocket performance, incident and reflected shocks, and Chapman-Jouguet detonations, Tech. Rep. SP-273, NASA (1971).

[70] R. J. Kee, F. M. Rupley, J. A. Miller, Chemkin-ii: a fortran chemical kinetics package for the analysis of gas-phase chemical kinetics, Tech. rep., Sandia National Laboratories (1991).

[71] T. Poinsot, D. Veynante, Theoretical and numerical combustion, R.T. Edwards, 2nd edition., 2005.

[72] J. Ely, H. J. M. Hanley, Prediction of transport properties. 2. Thermal conductivity of pure fluids and mixtures, Industrial \& Engineering Chemistry Fundamentals 22 (1) (1983) 90-97.

[73] S. Takahashi, Preparation of a generalized chart for the diffusion coefficients of gases at high pressures, journal of Chemical Engineering of Japan 7 (6) (1974) 417-420.

[74] R. Bird, W. Stewart, E. Lighfoot, Transport phenomena, John Wiley, New York, 1960.

[75] A. Jameson, W. Schmidt, E. Turkel, Numerical solution of the euler equations by finite volume methods using runge kutta time stepping schemes, in: 14th Fluid and Plasma Dynamics Conference, American Institute of Aeronautics and Astronautics, 1981, pp. 1259-1278. doi:10.2514/6. 1981-1259. 
[76] A. L. Gaitonde, A dual-time method for two-dimensional unsteady incompressible flow calculations, International journal for numerical methods in engineering 41 (6) (1998) 1153-1166.

[77] B. Leonard, A stable and accurate convective modelling procedure based on quadratic upstream interpolation, Computer Methods in Applied Mechanics and Engineering 19 (1) (1979) 59 -98. doi:http://dx.doi.org/ $10.1016 / 0045-7825(79) 90034-3$.

[78] R. C. Swanson, E. Turkel, On central-difference and upwind schemes, Journal of Computational Physics 101 (2) (1992) 292 - 306. doi:10.1016/ 0021-9991 (92) 90007-L.

[79] A. M. Ruiz, G. Lacaze, J. C. Oefelein, R. Mari, B. Cuenot, L. Selle, T. Poinsot, Numerical benchmark for high-reynolds-number supercritical flows with large density gradients, AIAA Journal 54 (5) (2015) 1445-1460. doi:10.2514/1. J053931.

[80] G. Sod, A survey of several finite difference methods for systems of nonlinear hyperbolic conservation laws, Journal of Computational Physics 27 (1) (1978) 1-31.

[81] R. Prosser, Improved boundary conditions for the direct numerical simulation of turbulent subsonic flows. I. Inviscid flows, Journal of Computational Physics 207 (2) (2005) 736-768.

[82] J. Oefelein, Large eddy simulation of turbulent combustion processes in propulsion and power systems, Progress in Aerospace Sciences 42 (1) (2006) $2-37$.

[83] G. J. Van Wylen, R. E. Sonntag, Fundamentals of classical thermodynamics, 3rd Edition, Wiley, New York, 1986.

[84] P. Roe, Approximate Riemann solvers, parameter vectors and difference schemes, Journal of Computational Physics 43 (1981) 357-372. 
[85] B. Van Leer, Towards the ultimate conservative difference scheme IV. A new approach to numerical convection, Journal of Computational Physics 23 (1977) 276-299.

[86] P. Jorgenson, E. Turkel, Central Difference TVD Schemes for Time Dependent and Steady State Problems, Journal of Computational Physics 107 (2) (1993) 297 - 308. doi:10.1006/jcph.1993.1145.

1060 [87] G. Lacaze, J. C. Oefelein, Modeling high-density-gradient flows at supercritical pressures, in: 49th AIAA/ASME/SAE/ASEE Joint Propulsion Conference, American Institute of Aeronautics and Astronautics, 2013, pp. 3717-3734. doi:10.2514/6.2013-3717. 